\title{
Large-scale 3D random polycrystals for the finite element method: Generation, meshing and remeshing
}

\author{
R. Quey ${ }^{\mathrm{a}, \mathrm{b}, *}$, P.R. Dawson ${ }^{\mathrm{a}}$, F. Barbe ${ }^{\mathrm{c}}$ \\ a Sibley School of Mechanical and Aerospace Engineering, Cornell University, Ithaca, NY 14853, USA \\ b École des Mines de Saint-Étienne, Centre SMS, CNRS UMR 5146, 158 Cours Fauriel, 42023 Saint-Étienne, Cedex 2, France \\ ' INSA Rouen, Groupe de Physique des Matériaux, CNRS UMR 6634, 76801 Saint Étienne du Rouvray, France
}

\begin{abstract}
A methodology is presented for the generation and meshing of large-scale three-dimensional random polycrystals. Voronoi tessellations are used and are shown to include morphological properties that make them particularly challenging to mesh with high element quality. Original approaches are presented to solve these problems: (i) "geometry regularization", which consists in removing the geometrical details of the polycrystal morphology, (ii) "multimeshing" which consists in using simultaneously several meshing algorithms to optimize mesh quality, and (iii) remeshing, by which a new mesh is constructed over a deformed mesh and the state variables are transported, for large strain applications. Detailed statistical analyses are conducted on the polycrystal morphology and mesh quality. The results are mainly illustrated by the high-quality meshing of polycrystals with large number of grains (up to $10^{5}$ ), and the finite element method simulation of a plane strain compression of $\varepsilon=1.4$ of a 3000-grain polycrystal. The presented algorithms are implemented and distributed in a free (open-source) software package: Neper.
\end{abstract}

\section{Introduction}

Many important phenomena occurring in polycrystalline materials depend on the mechanical fields that develop at the scale of the microstructure. One can cite, for example, fatigue, rupture, plasticity-environment interactions (stress corrosion cracking), microtexture development at large strains or the nucleation of phase transformations. It is generally recognized that the local mechanical fields depend on several microstructural factors: the single crystal behavior, the distribution of grain orientations, and the polycrystal morphology. While the first two factors mainly control the global behaviors, for example the macroscopic stress-strain response or deformation texture development, a representative polycrystal morphology is generally required for the prediction of local phenomena.

In the last decade, several investigations have been reported on the 3D experimental characterization of real polycrystal morphologies [1-3]. They have provided important information such as the distribution of grain size, the number of first neighbors, and grain boundary curvatures. Because the methods are lengthy and expensive, they have been applied only to a few, relatively small samples, made of $10^{2}$ to $10^{3}$ grains.

* Corresponding author. Present address: CEA-Leti, MINATEC Campus, F38054 Grenoble, France.

E-mail addresses: rq27@cornell.edu (R. Quey),prd5@cornell.edu (P.R. Dawson) fabrice.barbe@insa-rouen.fr (F. Barbe).
In parallel, computational methods, notably the finite element method (FEM), have been developed to simulate the mechanical behavior of virtual polycrystals (see for example [4-7]). Many of the studies based on 3D polycrystals have defined grains using regular morphologies that are straightforward to mesh, such as cubes, dodecahedra or truncated octahedra [8-11]. The mechanical fields that develop in the microstructure nevertheless appear very complex. These results motivate not only the use of polycrystals with larger numbers of grains, but also more representative grain morphologies. Notably, an important step is to account for the inherent variability in grain size and shape. Numerous applications can take advantage of such improvements. Local phenomena such as crack instantiation and propagation in brittle materials or the nucleation of phase transformations depend on the maxima of the mechanical fields. The size of the statistically representative volume element needed for their prediction (in terms of the number of grains) is much larger than for determining average macroscopic properties. Such is also the case for the prediction of stress and strain localization patterns. In particular, determining correct characteristics of plastic strain bands will require of the order of $10^{4}$ grains, because such bands can expand over several grains in a given direction [12]. Similarly, low crystal-symmetry or multiphase materials, which inherently show large variabilities of the mechanical fields, require large numbers of grains for their global and local properties to be determined accurately [13-15]. These realities argue for even larger virtual polycrystals as well as ones that are more representative of the features of the microstructures. 
Several authors have proposed analytical and numerical methods to construct random polycrystal morphologies. These methods can be rooted in the basic principles of phase transformations (leading in some cases to Voronoi tessellations) [6,16], physically-driven simulations of annealing or recrystallization [17-19], or algorithms that attempt to directly reproduce statistical data coming from experimental characterizations [20]. To simulate the deformation of such polycrystals, a number of approaches have been reported that involve representing the microstructure on a grid, including a finite element method with the mapped meshing technique [7,14], fast Fourier transforms [21], and a level-set approach [19]. The drawback of such simplified meshing schemes is that the interfaces between grains (grain boundaries, triple lines and quadruple points) are poorly described. The resulting strain and stress fields may be affected, at least in the immediate vicinity of the interfacial features. This can lead to strong artefacts in the prediction of grain-scale surface roughening [22], grain boundary sliding in nanocrystalline materials [23] or in local micromechanical approaches of fracture in bainite [24]. Furthermore, more complex applications, such as stress corrosion cracking associated with the diffusion of species at grain boundaries [25] cannot be addressed adequately without a conforming mesh. Direct, free meshing algorithms (also called "unstructured meshing") in principle can render a conforming mesh for any polycrystal morphology, but doing so have proven to be difficult to implement for random morphologies.

Among randomly generated morphologies, Voronoi tessellations have the advantages of being defined analytically and having straight triple lines and flat grain boundaries [26]. Voronoi tessellations are sometimes considered as coarse models of microstructures [2], because they quite significantly underestimate the variability in grain size and overestimate the number of grain first neighbors. (It will be shown in Section 2.1.2 that this can be compensated by correction schemes.) Still, Voronoi tessellations show important variabilities in grain size and shape which are representative of real polycrystal morphologies, but that make them particularly challenging to mesh with good-quality elements. Consequently, free meshing of Voronoi tessellations has been applied almost exclusively to relatively small polycrystals $\left(<10^{3}\right.$ grains) and used primarily in the context of small deformation studies ( $\lesssim 1 \%$ ) $[27,14,25,28,24]$. In such configurations, element quality is not as critical an issue as when the number of grains becomes large or deformation severe.

In this article, a methodology is presented for generating viable meshes for "large-scale" Voronoi tessellations, comprising several thousand of grains. The aim is to obtain meshes that are comprised of high-quality elements and are without regions that are overly or inadequately resolved. Specifically, the elements must be equiaxed in shape and uniform in size. Inevitable issues that affect the meshing of large-scale Voronoi tessellations are outlined in Section 2, together with methods to resolve these issues. The resulting meshes are suitable for small-strain as well as large-strain studies $(\varepsilon \simeq 0.5)$. A remeshing strategy is also presented, which facilitates reaching strains of between 1 and 2 . The robustness of the random polycrystal generation and meshing methodology is illustrated by their application to the prediction of deformation textures and microtextures of an aluminium alloy (Section 3).

\section{Polycrystal generation}

The generation of a polycrystal mesh involves at least two steps: (i) the generation of the polycrystal morphology, described by sets of points, lines, surfaces and volumes, and (ii) the free meshing of the morphology. An additional step, the "remeshing", offers the possibility to reach higher deformations by reconstructing a high-quality mesh from a deformed mesh comprising poor-quality elements.

\subsection{Polycrystal morphology}

\subsubsection{Voronoi tessellation}

Mathematically, a Voronoi tessellation of an $n$-D space is a collection of $n$-D entities that fills the space with no overlaps and no gaps. These entities are polyhedra and are formally defined as zones of influence of a particular set of points, corresponding to their centres. Being given (i) a spatial domain $D \in \mathfrak{R}^{n}$, (ii) a set of points $E=\left\{G_{i}\left(\underline{\mathbf{x}}_{i}\right)\right\}$ within $D$, and (iii) a norm $d(\bullet, \bullet)$, every point $G_{i}$ is associated a Voronoi polyhedron $C_{i}$ as follows,

$C_{i}=\left\{P(\underline{\mathbf{x}}) \in D \mid d\left(P, G_{i}\right)<d\left(P, G_{j}\right) \quad \forall j \neq i\right\}$,

In the present work, the dimension $n$ is equal to 3 , the norm $d$ is taken as the Euclidean distance, and the set of points $E$ is considered to be randomly distributed. In such a case, the tessellations are sometimes referred to as "Poisson-Voronoi tessellations". By construction, a Voronoi polyhedron is convex; hence the intersection of two Voronoi polyhedra is a plane, called "tessellation face", the intersection of three Voronoi polyhedra is a straight line, called "tessellation edge", and the intersection of four Voronoi polyhedra is a point, called "tessellation vertex". From a physical point of view, the generation of Voronoi tessellations corresponds to a process of solidification or recrystallization where all grains nucleate at the same time and grow isotropically at the same rate. An example of a Voronoi tessellation is given in Fig. 1. Voronoi tessellations qualitatively reproduce the first-order properties of real polycrystal morphologies: the distribution of grain size and the number of first neighbors, as illustrated in Fig. 2.

The free-meshing technique draws on other statistical properties: the edge length and face diameter distributions, provided in Fig. 3. The distributions show that Voronoi tessellations include large numbers of relatively small entities (edges and faces). Such geometrical details are particularly problematic for meshing because their lengths can be smaller than the target characteristic length of the mesh elements. This results in a mesh that is overly refined in the vicinity of those details relative to the desired target (illustrations will be provided in Fig. 8). Without such refinement, however, element quality suffers. Thus, a high degree of refinement is necessary to assure convergence even though increased refinement has little if any impact on the predictions. For classical configurations, the mesh overrefinements can increase the total number of elements by a factor of 2 , and as a consequence significantly increase the computational resources needed (see Fig. 8).

\subsubsection{Regularization}

The Voronoi tessellations must be modified to be suitable for a proper free meshing. In this section, an original approach called "regularization" is proposed, which consists in removing the small entities (edges and faces). This idea has been suggested by Nygårds and Gudmundson in 2002 [27].

The main principle of the geometry regularization is illustrated in Fig. 4. It consists of removing the "small edges", whose length is below a given threshold value. That corresponds to the maximum length needed to avoid an overly refined mesh. The small edges of a 9-grain polycrystal are highlighted on Fig. 4 (a). In the present case, the threshold limit is set to 0.13 for grains of unit volume. Deleting a small edge consists of replacing it and its two connected vertices by a single vertex. This leads to topological modifications of the tessellation: more than four grains may intersect at a single vertex and more than three faces may intersect at a single edge. Moreover, replacing two vertices by a single one results in distortions of the faces to which it belongs: the new vertex has a position 


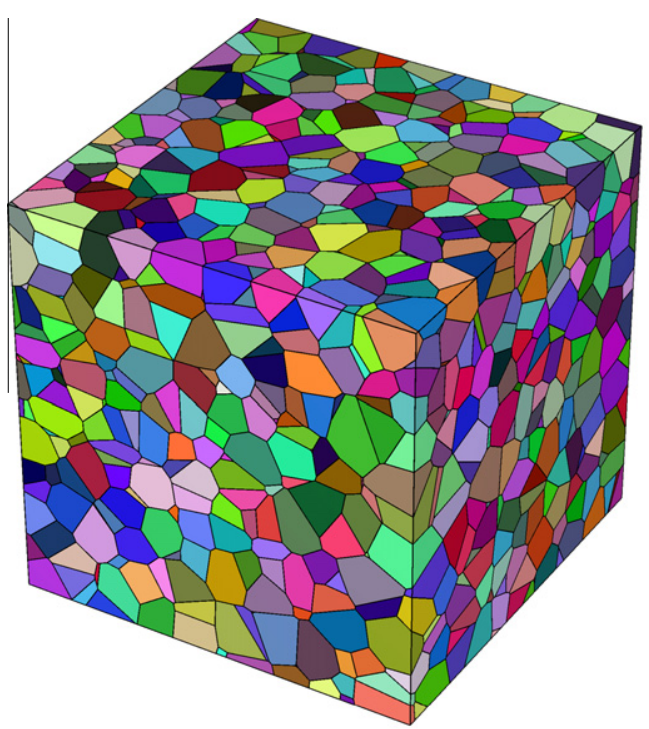

a

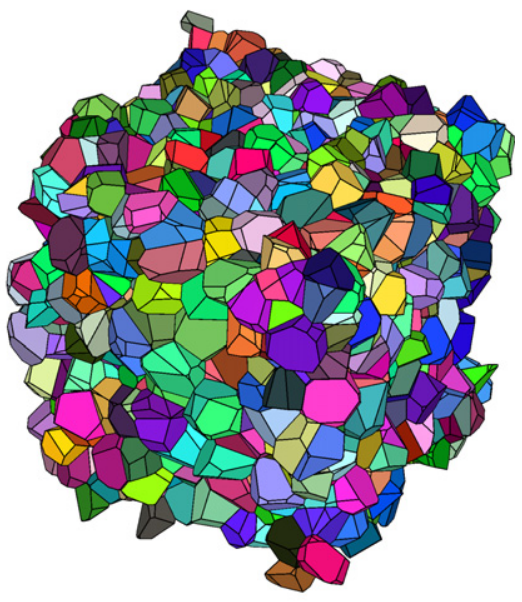

b

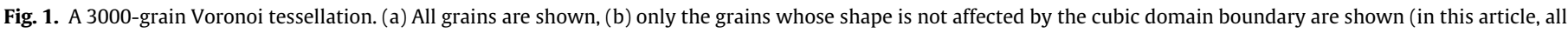
statistics are provided for such grains).
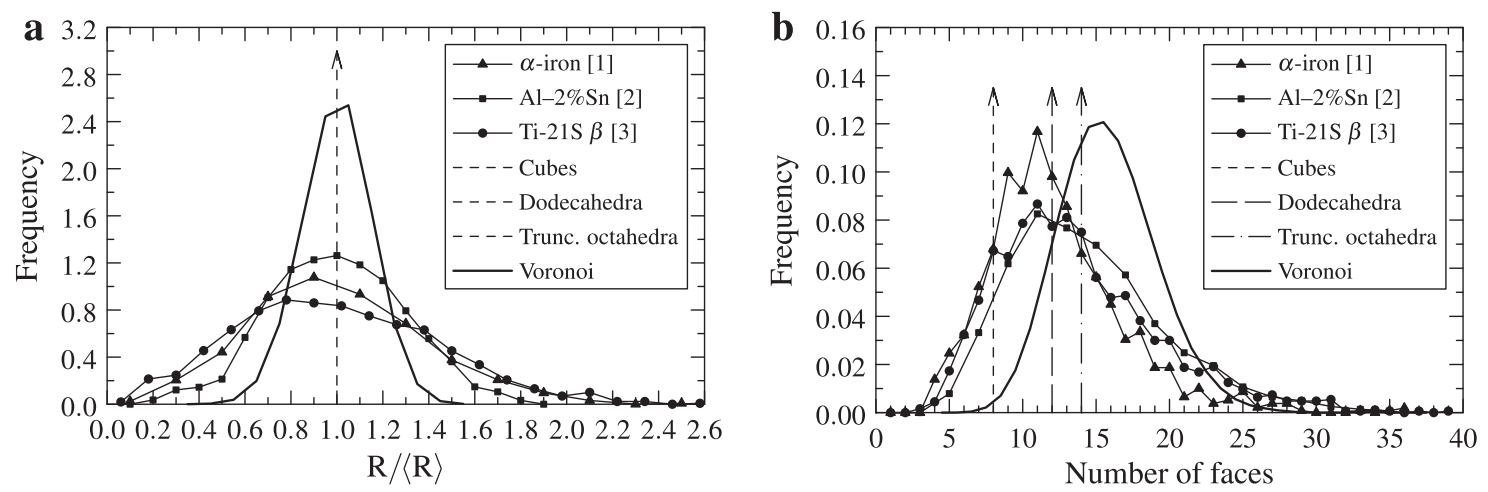

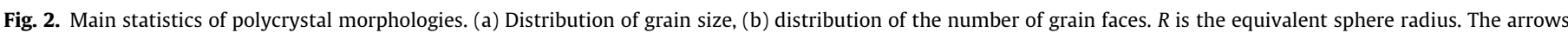
denote Dirac-type distributions.
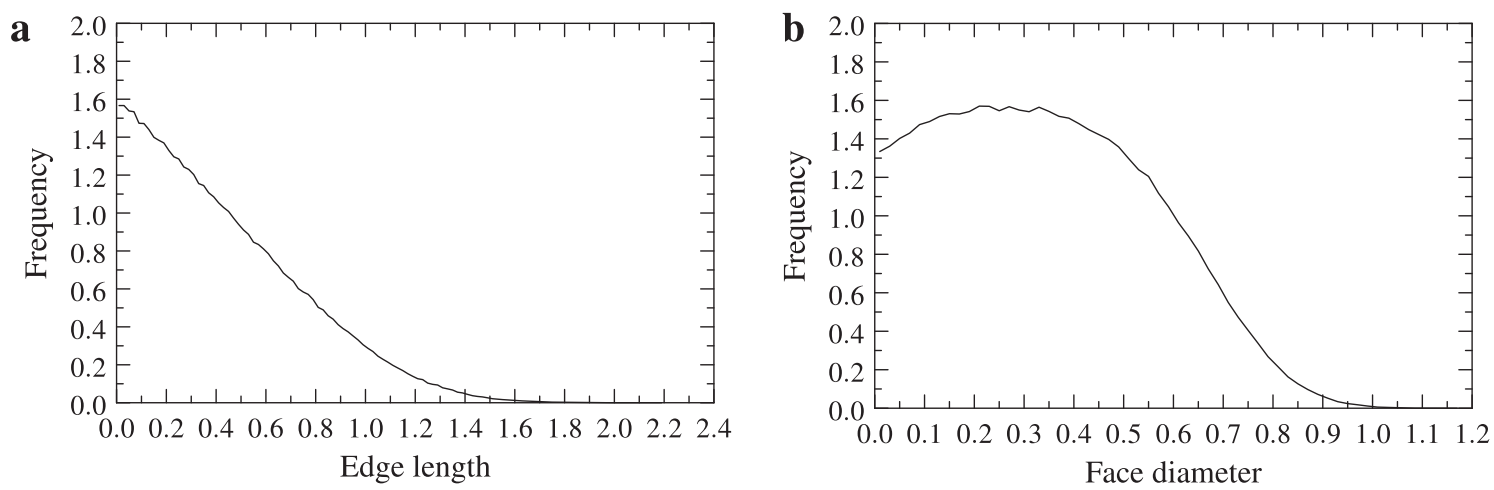

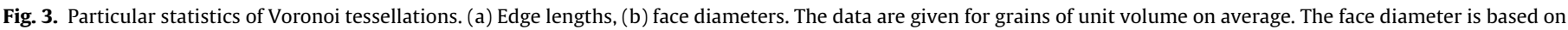
the equivalent circle. Note the large number of small entities. Data collected on $10^{5}$ grains.

different from those of its two parent vertices, and as a consequence does not lie within the initial planes of its faces. Thus, such a face has vertices and edges that are not within its initial plane and that actually do not have any common plane. The face boundary is still well defined from its set of straight edges, but its interior is not and requires modification (see Fig. 4).
The different steps involved in an edge deletion are designed to minimize the local geometric distortions. The steps are:

2.1.2.1. New vertex positioning. The new vertex is positioned so as to minimize the distances to the initial face planes. The following quantity is minimized (least-square criterion): 

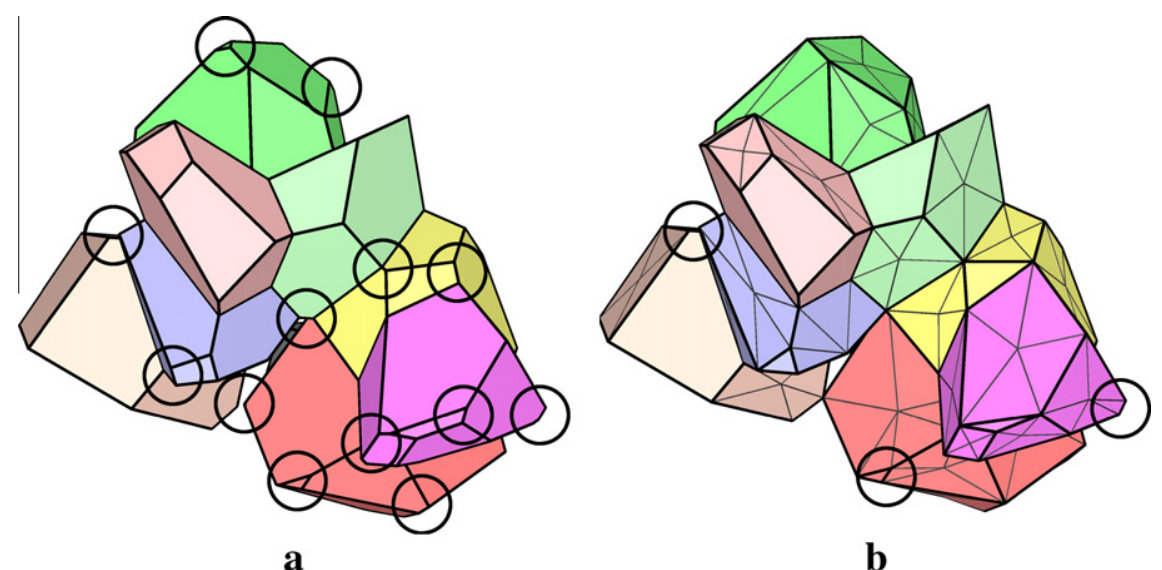

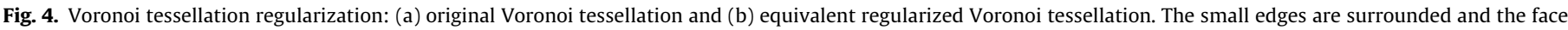
interpolations triangles are marked in gray. Case of a 9-grain polycrystal.

$d=\sqrt{\sum_{i=1}^{n_{f}} d_{i}^{2}}$,

where $d_{i}$ is the distance from the vertex to the initial plane of face $i$, and $n_{f}$ the number of faces of the vertex.

2.1.2.2. New vertex face interpolation. Each vertex face is divided into a set of triangles, which are based on the face edges, and a "master" point, which can be for example the face barycentre. The triangles are shown through gray lines on Fig. 4 (b). The face distortion is quantified by the maximum angle between the normals of any two of the triangular parts of the face and is referred to as the face flatness fault, $f$. This angle must remain relatively small to minimize the grain shape changes and can vary depending on the position of the master point. In practice, several positions are considered: the face barycentre and the face vertices. The position for which the face flatness fault is the smallest is retained.

The geometry distortion resulting from the edge deletion is then quantified by the maximum of the flatness faults of the new vertex faces. If its value is below a given threshold value (typically $20^{\circ}$ ), the edge deletion is accepted. In practice, the values frequently are higher than the threshold value when the new vertex itself belongs to a small edge. In such a case, before the edge deletion is rejected, the deletion process also is applied to the new vertex small edges. The case having lower distortion is retained. If the distortion is reduced below the threshold value, the two edges are deleted. This process is applied to all the small edges, from the smallest to the largest. As the local configurations may change, several loops may be applied to delete as many small edges as possible. This geometry regularization algorithm is also applicable to periodic Voronoi tessellations which are often used as means to reduce the size of the representative volume element $[28,29]$, and more particularly when the tessellation is not cut by the domain boundary [29].

The distributions of edge lengths and face diameters obtained after geometry regularization are given in Fig. 5. As the allowed maximum flatness fault is increased, more and more small edges are deleted, and convergence is observed from a value of about $20^{\circ}$. It should also be noted that small faces are deleted as a consequence of deleting small edges. This can be explained by the fact that they are mostly made of small edges. An additional change of the polycrystal morphology concerns the distribution of face flatness faults (see Fig. 6), which now spread between 0 and $20^{\circ}$ (it should be noted, however, that $90 \%$ of them are smaller than $6^{\circ}$ ). The influence of geometry regularization on the distributions of grain size and the number of grain faces are illustrated in Fig. 7 for different values of the maximal length for which an edge can be deleted $\left(l_{\max }\right)$. Grain size is almost unchanged by geometry regularization. On the other hand, the distribution of the number of grain faces is significantly modified. For a value of $l_{\max }=0.13$ for grains of unit volume (case of Figs. 4 and 5), the average number of faces per grain, which is also the number of first neighbors, is decreased from 15.1 to 13.7 (the average number of edges per grain is decreased from 39.1 to 31.8 ). As $l_{\max }$ increases, the number of grain faces decreases. For $l_{\max }=0.26$, the average number of grain faces is
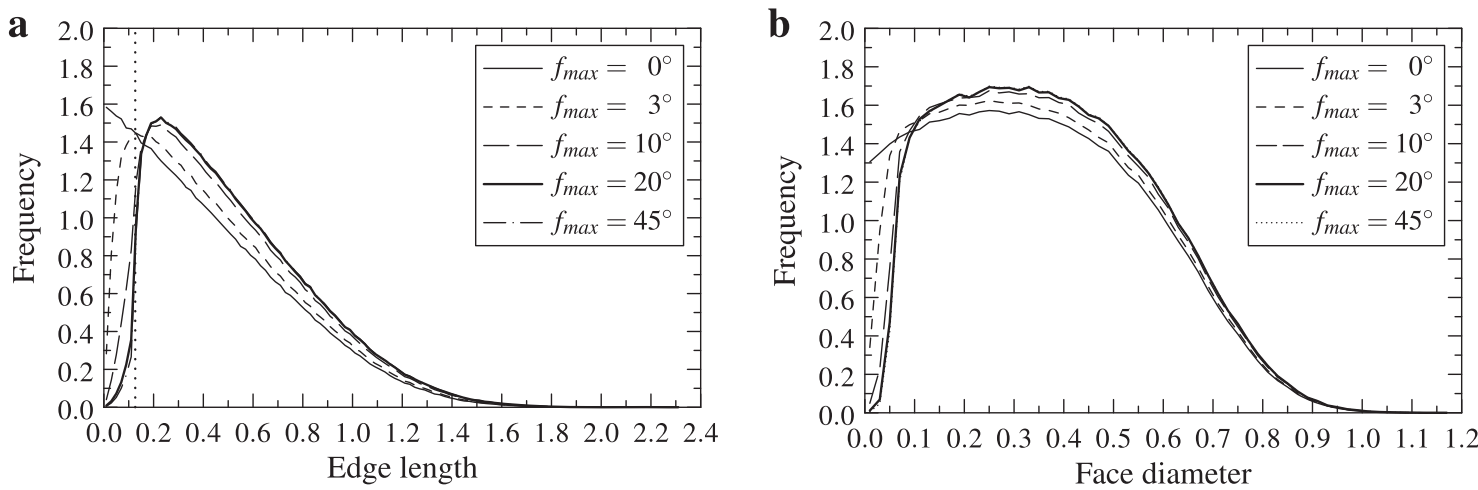

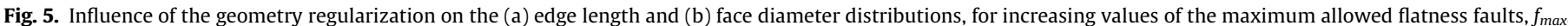

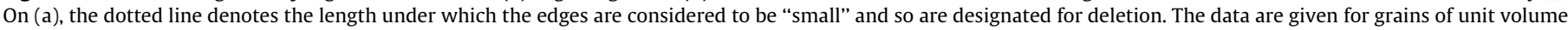
on average. The face diameter is based on the equivalent circle. Data collected on $10^{5}$ grains. 


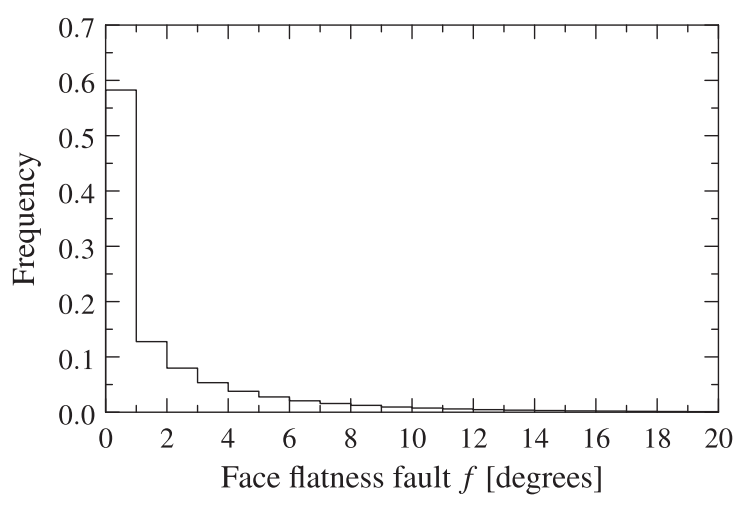

Fig. 6. Influence of the geometry regularization on the face flatness faults, for a maximum allowed value of $f_{\max }=20^{\circ}$. Note that most of the angles are much smaller. Data collected on $10^{5}$ grains (all faces).

12.3, which provides a good agreement with experimental values (Fig. 2).

\subsection{Meshing strategies}

Voronoi tessellations (in their standard or regularized forms) show an infinite variety of grain morphologies; a grain has a unique shape, with between 4 and 36 faces and each face can have between 3 and 16 edges [30]. Moreover, in some simulation configurations (for example [12]), one may want to use relatively few elements per grain, which highly constraints the meshing process. Generating a mesh of high-quality elements of sizes as close as possible to a target value requires well-defined and robust algorithms, which are detailed in the present section.

\subsubsection{Meshing}

The meshing approach is based on a widely-utilized bottom-up flow that consists in meshing the 0D, 1D, 2D, and 3D entities (the tessellation vertices, edges, faces, and polyhedra), successively. The $n$-D mesh generation is constrained by the $(n-1)-\mathrm{D}$ mesh and the element lengths are interpolated from it. Here, we also explicitly consider the target mesh characteristic length to ensure mesh size homogeneity to the greatest extent possible. Each entity is meshed independently of the other entities of same dimension.

For a given polycrystal morphology, two quantities are considered for the meshing: the target characteristic length, $c_{l}$, and a tolerance for its spatial evolution, called the progression factor, $p_{l}$. This value is the maximum ratio between the lengths of two adjacent elements and is used for the 0D and 1D meshings. The meshing is applied as follow (examples are shown in Fig. 8):

2.2.1.1. OD meshing. Each vertex is given a OD element, which contains only one node. The node is attributed a characteristic length as follows: if the vertex does not have small edges, the node characteristic length takes the value of $c_{l}$, otherwise, it is set to the length of the smallest edge multiplied by $p_{l}$.

2.2.1.2. 1D meshing. Each edge is meshed into 1D elements. The element lengths are derived from the value at the two vertices and $c_{l}$. If the vertex characteristic lengths are equal to $c_{l}$, the edge is divided into elements of length $c_{l}$. If they are smaller than $c_{l}$, then the element lengths start from these values at the vertices and are progressively increased by a factor $p_{l}$ until they reach $c_{l}$ (in the body of the edge). Then, each new node is assigned a characteristic length which will be used for $2 \mathrm{D}$ meshing, and is equal to the length of its longest element.

2.2.1.3. $2 D$ and $3 D$ meshings. Each face is meshed into triangle elements. As for the 1D meshing, the element lengths are derived from the characteristic lengths of the node of the boundary mesh, together with the target value $c_{l}$. The same process is applied for the 3D meshing, each polyhedron being discretized into tetrahedral elements. For both 2D and 3D meshing, external meshing libraries are used: the Gmsh and Netgen packages [31,32]. Gmsh has been used previously for crystal plasticity studies [33]. The main principle of the employed algorithms is to produce a mesh with elements of as high quality as possible and of size as close as possible to the target size, $c_{l}$. Further details can be found in Ref. [31].

In the case of a regularized tessellation, the faces are generally not defined in a single plane, which is, however, mandatory for 2D meshing. A preliminary step is thus to project orthogonally such faces in a plane. For each face, the plane normal is defined as,

$\underline{\mathbf{n}}=\frac{\sum_{i=1}^{n_{t}} A_{i} \underline{\mathbf{n}}_{i}}{\left|\sum_{i=1}^{n_{t}} A_{i} \underline{\mathbf{n}}_{i}\right|}$,

where $A_{i}$ and $\mathbf{n}_{i}$ are the area and normal of the $i$ th triangle part of the face. $n_{t}$ is the number of triangle parts. $|\underline{\mathbf{x}}|$ denotes the norm of $\underline{\mathbf{x}}$. The face is then meshed in that plane. The last step is to project the mesh back along the same direction, $\underline{\mathbf{n}}$, onto the face interpolation triangles. It should be noted that, although the interpolation triangles could be used to facilitate the meshing process, this would introduce new 1D mesh constraints, which in turn would decrease element quality.
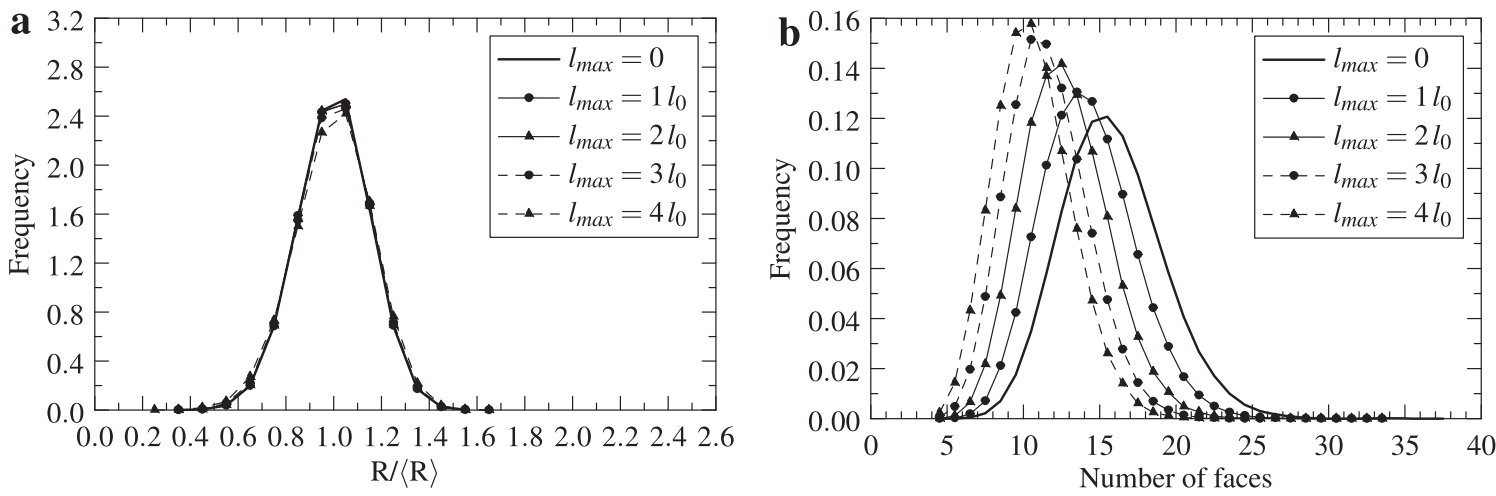

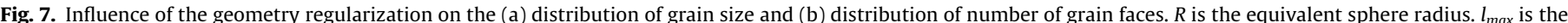

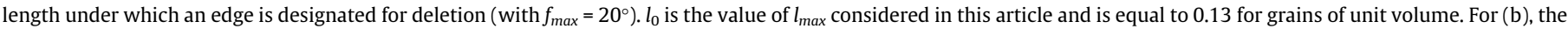
average values are $15.1,13.7,12.3,11.1$ and 10.2 , respectively. Data collected on $10^{5}$ grains. 


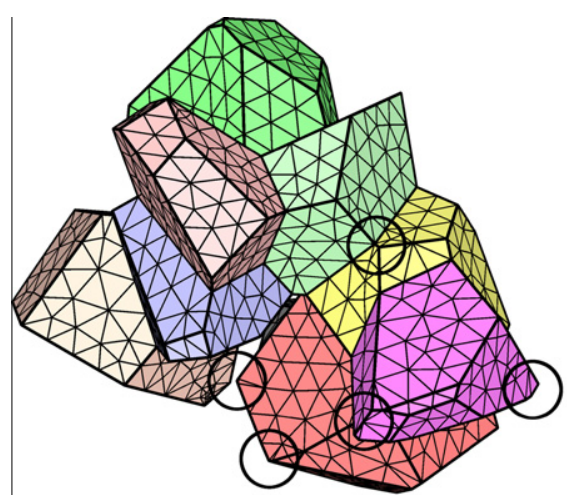

a

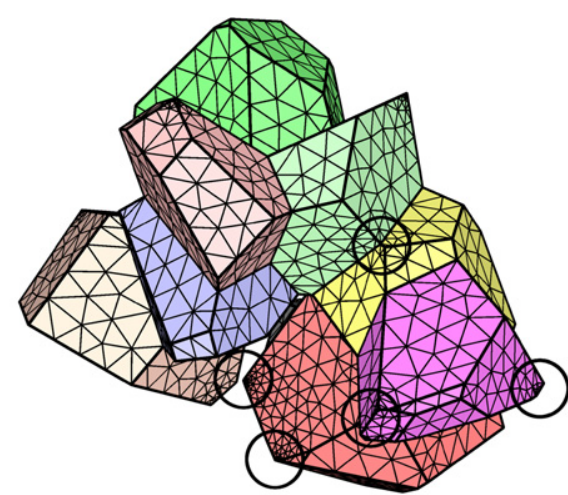

b

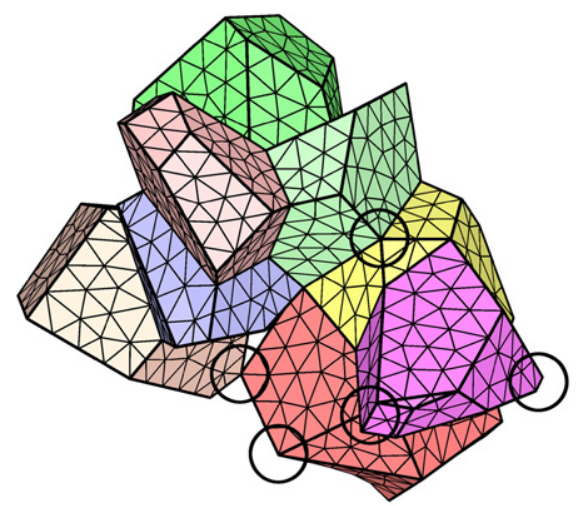

c

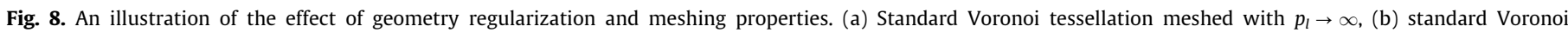

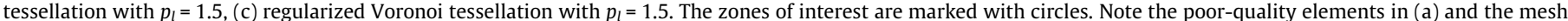
overrefinements in (b) near the small edges; (c) shows high-quality elements and no mesh overrefinements.
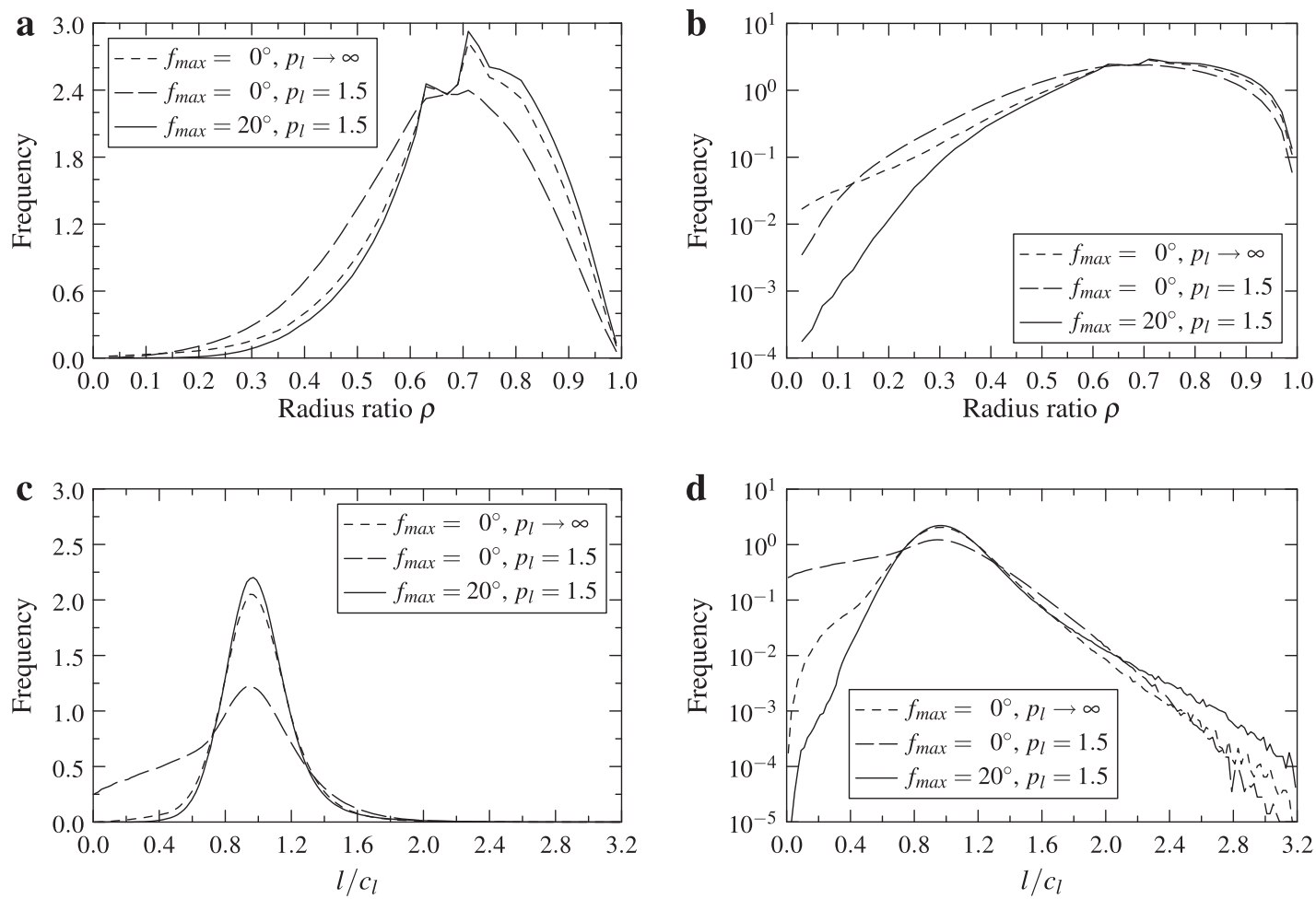

Fig. 9. Mesh quality: (a-b) element qualities and (c-d) relative element sizes for different meshing strategies. Data collected on $10^{5}$ grains. 

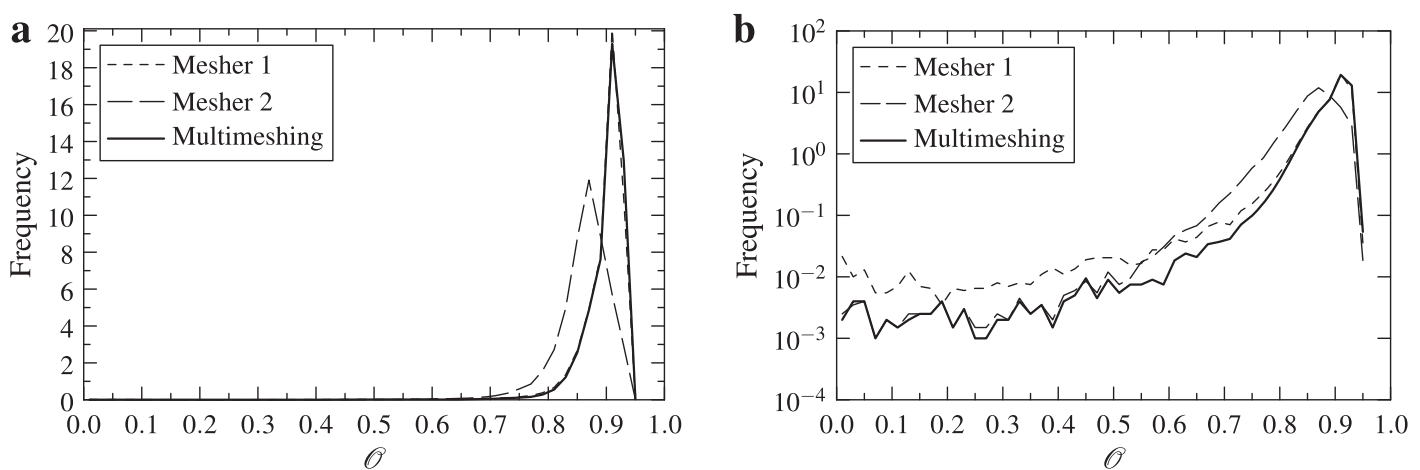

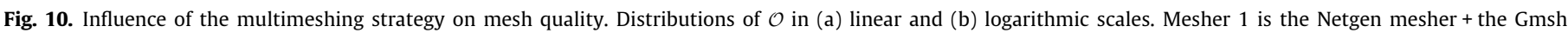
optimization; mesher 2 is the Netgen mesher + both the Netgen and Gmsh optimizations. Data collected on $10^{5}$ grains.

In this study and unless stated otherwise, the value of $c_{l}$ is held constant and equal to 0.25 for grains of unit volume (which leads to about 600 elements per grain for regularized morphologies). We focus on the effect of the progression factor, $p_{l}$, and the geometry regularization, as illustrated in Fig. 8. As stated previously, for standard Voronoi tessellations, high values of $p_{l}$ will lead to a mesh quite homogeneous in size, but with elements of low quality near the small edges (Fig. 8 (a)). In contrast, a typical value of $p_{l}$ of 1.5-2 avoids poor-quality elements, but severely over-refines the mesh around the small edges (Fig. 8 (b)). The improvements associated to regularized Voronoi tessellations are illustrated on Fig. 8 (c). With a proper value of $p_{l}(1.5-2)$, the mesh does not possess poor-quality elements nor is it overly refined locally. The corresponding statistical data on element quality and element size are given in Fig. 9. In this article, the element quality is quantified by the radius ratio, a common measure that goes from 0 for poorquality (flat or elongated) elements to 1 for high-quality (equilateral) elements [31]. The element size is quantified by the average of the element edge lengths. It is shown that the geometry regularization results in a much better mesh, in terms of both element quality and element size distributions. It should be noted that, on Fig. 9, both linear and logarithmic scales are provided for the distributions. Although most of the elements are of high quality (Fig. 9 (a)), there are some elements of very poor quality (Fig. 9 (b)). This is important since such elements limit the level of deformation that can be applied to the mesh before the first degenerate element appears.

\subsubsection{Multimeshing}

The infinite variety of grain shapes provided by Voronoi tessellations makes them challenging for the meshing software packages to provide high-quality meshes in a systematic way. Moreover, in some configurations, a given meshing software package simply may fail. Even for regularized Voronoi tessellations, a single meshing algorithm can return some number of poor-quality elements, as can be seen on Fig. 9. Free meshing methods typically involve two steps: a preliminary meshing and mesh optimization, during which topological operations are applied to improve element quality [31,32,34,35]. As pointed out in Ref. [31], the topological operations are well-known (element swapping and smoothing), but there is no known best way to combine them. From the user point of view, several algorithms and software packages are available, each one being able to provide the best-quality mesh in specific situations.

The proposed approach, called multimeshing, consists of using simultaneously several meshing algorithms. It is applied to the 2D and 3D meshings in similar ways, so only the 3D case will be detailed in the following. The principle is that, for each polyhedron of the tessellation, a mesh is generated from each meshing algorithm and the best one is retained. This has the benefit of generating the best mesh for each polyhedron and optimizes the polycrystal mesh quality subject to the limitations of the suite of meshing codes.

To apply this method, the quality of a polyhedron mesh must be quantified. A criterion, $\mathcal{O}$, is introduced to account for both the element distortions and the element sizes,

$\mathcal{O}=f\left(\mathcal{O}_{\text {dis }}, \mathcal{O}_{\text {size }}\right)$.

We choose a function of the form:

$\mathcal{O}=\mathcal{O}_{\text {dis }}^{1-\alpha} \mathcal{O}_{\text {size }}^{\alpha}$

with $\alpha \in[0,1]$. Low values of $\alpha$ favor low element distortions while high values favor high element size homogeneity. A value of $\alpha=0.2$ provides an effective balance.

For $\mathcal{O}_{\text {dis }}$, we choose a function of the form,

$\mathcal{O}_{\text {dis }}=\prod_{i=1}^{n_{e}} \sigma_{i} \quad$ where $\left\{\begin{array}{ll}\text { if } \rho_{i}<1, & \sigma_{i}=\rho_{i}{ }^{\exp \left(\frac{\rho_{i}^{\beta}}{\rho_{i}^{\beta}-1}\right)} \\ \text { if } \rho_{i}=1, & \sigma_{i}=1,\end{array}\right.$ with $\beta=0.1$,

where $\rho_{i}$ is the radius ratio of element $i$ and $n_{e}$ is the number of elements. $\mathcal{O}_{\text {dis }}$ is defined on the interval $[0,1]$, where high values mean low element distortions. This expression of $\sigma_{i}$ produces values very close to 1 for acceptable values of $\rho_{i}\left(\rho_{i} \in[0.1,1]\right)$ and small values if the mesh includes poor-quality elements (low $\rho_{i}$ ). The multiplicative form of $\mathcal{O}_{\text {dis }}$ penalizes a mesh more heavily as the number of poor-quality elements accumulates.

For $\mathcal{O}_{\text {size }}$, we choose a function of the form,

$\mathcal{O}_{\text {size }}=\left(\frac{1}{n_{e}} \sum_{i=1}^{n_{e}} v_{i}\right)^{3}$ where $\begin{cases}\text { if } l_{i}<c_{l}, & v_{i}=l_{i} / c_{l}, \\ \text { if } l_{i} \geqslant c_{l}, & v_{i}=c_{l} / l_{i},\end{cases}$

where $l_{i}$ is the equivalent length of element $i$ (taken as the average of the element edge lengths). $\mathcal{O}_{\text {size }}$ is defined on the interval $[0,1]$, where high values mean high element size homogeneity. The effect of multimeshing on $\mathcal{O}$ is illustrated on Fig. 10. Two meshing algorithms are used: the Netgen mesher [32] + the Gmsh optimization [31] and the Netgen mesher + both the Netgen and Gmsh optimizations (these combinations are also considered in Ref. [31]). For convenience, they will be considered as two distinct algorithms (referred to as "mesher 1" and "mesher 2", respectively). From Fig. 10 and for the considered mesh refinement level, it should be noticed that mesher 1 provides on average lower values of $\mathcal{O}$ than mesher 2, but also fewer poor-quality elements by nearly an order of magnitude. In other words, mesher 1 is less efficient on average, 

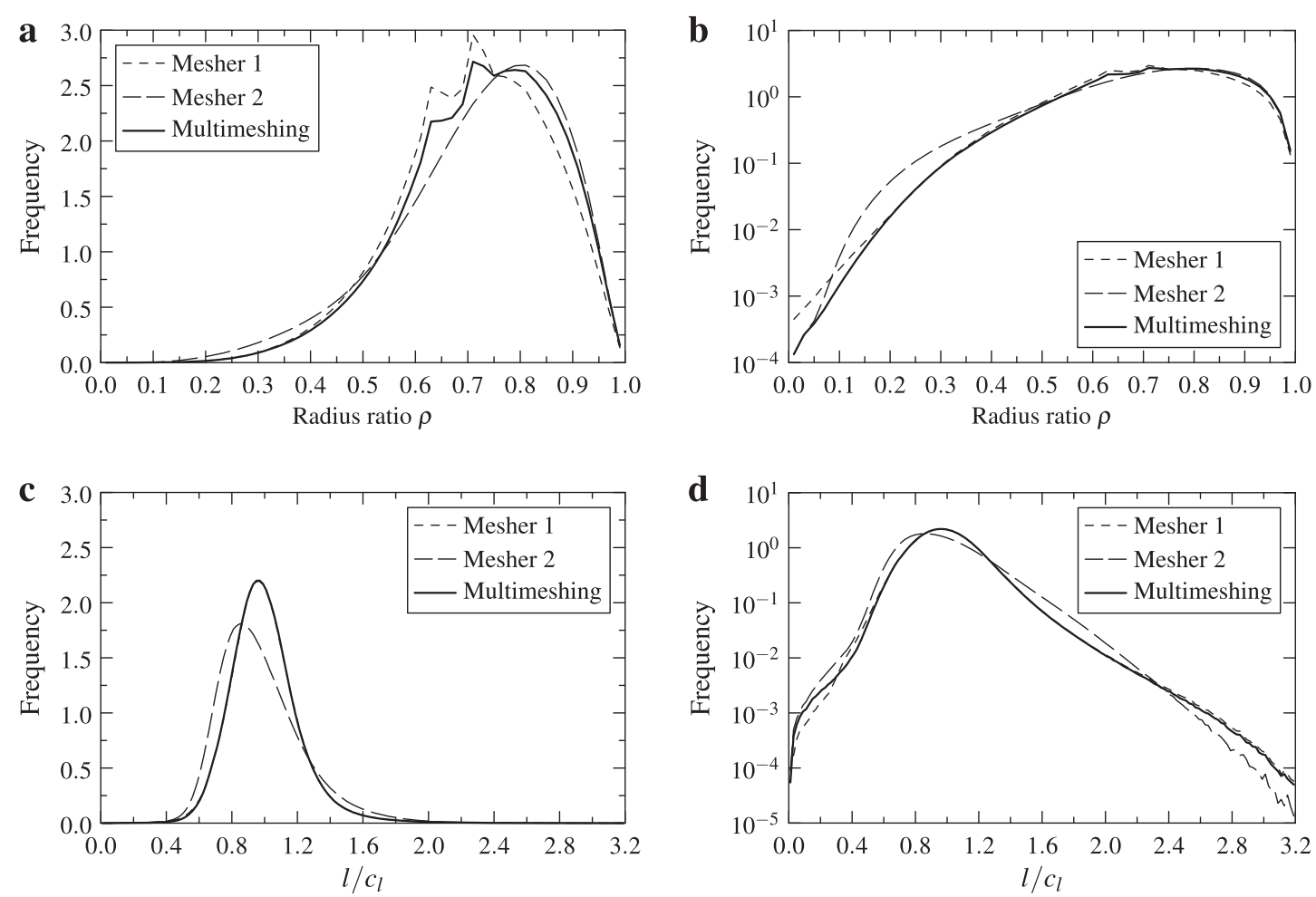

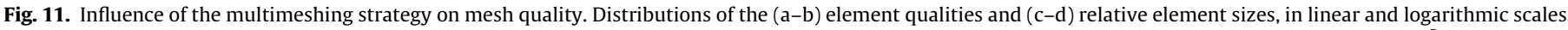
Mesher 1 is the Netgen mesher + the Gmsh optimization; mesher 2 is the Netgen mesher + both the Netgen and Gmsh optimizations. Data collected on $10^{5}$ grains.

but is much more effective for the more problematic cases. The multimeshing distribution selects the mesher 2 distribution at high-quality levels and the mesher 1 distribution at low-quality levels and, as a result, provides a final mesh more favorable than the ones provided by the two meshers taken individually. The effect of multimeshing on the element quality and size distributions is illustrated in Fig. 11. As expected, the multimeshing strategy provides better distributions than those of the meshers taken individually, which is especially the case at low element quality levels. These statistical measures indicate that a relatively low number of poor-quality elements will exist in the final mesh. For example, the proportion of elements with $\rho<0.01$ is about 1 per million and the proportion of elements with $\rho<0.05$ is approximately 510 per million. In practice, the resulting meshes are suitable for large-strain studies of large-scale polycrystals, as will be illustrated in Section 3. Strains of the order 0.5 can be applied to polycrystals with $10^{3}$ or $10^{4}$ grains (with $10^{6}$ elements or more), with few poor-quality elements present to cause early element degeneracies.

\subsection{Remeshing}

Applications such as industrial forming operations involve strains of the order 1-2 that are not attainable with a single polycrystal mesh. Even with an initial mesh of perfect quality, the globally imposed shape change together with the additional strain localizations would result in a degenerated element appearing before the target strain is attained. The maximum strain that can be imposed depends on the material properties, the type of deformation, and the polycrystal morphology, but can reasonably be considered of the order 0.7 [10]. To reach higher deformations, the mesh must be reconstructed, which is well-known as "remeshing". This involves the generation of a new mesh and the transfer of the nodal and elemental variables of the old, deformed mesh onto the new mesh (see Fig. 12).
The generation of a new mesh from an old mesh is based on the same rules as for the meshing of an undeformed Voronoi tessellation (Section 2.2.1). It is also applied in a bottom-up flow, from the OD to the 3D meshing, with the same two parameters controlling the mesh size: $c_{l}$ and $p_{l}$. The difference is that the edges, faces and polyhedra are now described by their respective deformed meshes (the tessellation topology is not changed). The remeshing is applied as follow:

\subsubsection{D remeshing}

Each vertex is assigned an element which contains one node only. The node is assigned a characteristic length in the same way as was done for the OD meshing.

\subsection{2. $1 D$ remeshing}

The polycrystal edges no longer are straight, which is mandatory for 1D meshing. So, the equivalent straight configuration is constructed where the nodes are placed according to their curvilinear coordinates in the deformed configuration. The straight configuration is meshed and the new mesh is mapped to the initial configuration through simple geometrical considerations. This ensures a proper description of the edge.

\subsection{3. $2 D$ and $3 D$ remeshings}

The 2D remeshing involves the same steps as the 2D meshing of non-plane faces: a projection in a mean plane, the meshing in that plane, and the projection back to the face. The difference is that the interpolation of the face is not defined by the triangle parts (see Section 2.2.1), but by the old face mesh. The 3D remeshing is applied in the same way as the 3D meshing.

For very deformed polycrystals, problematic configurations sometimes appear, which cannot be handled by the rules defined previously. First, some faces can be very distorted, so that it is impossible to project them onto a plane for 2D meshing without 


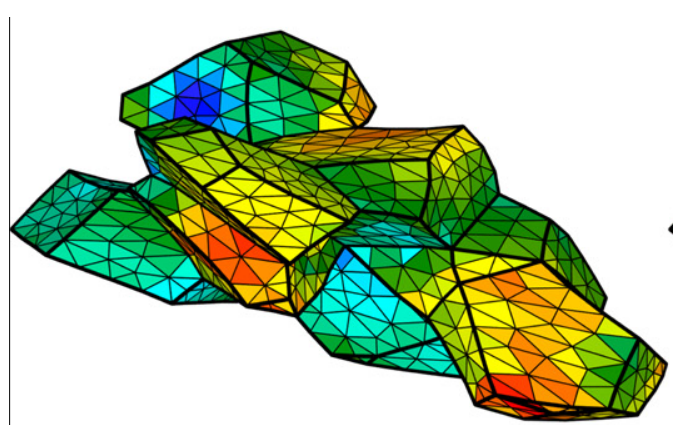

$\mathbf{a}$

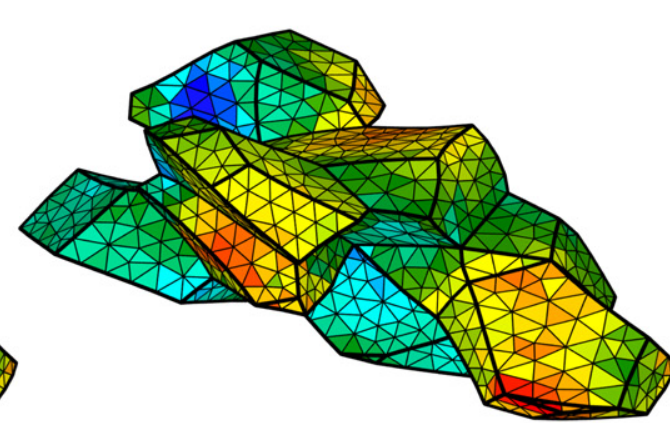

b
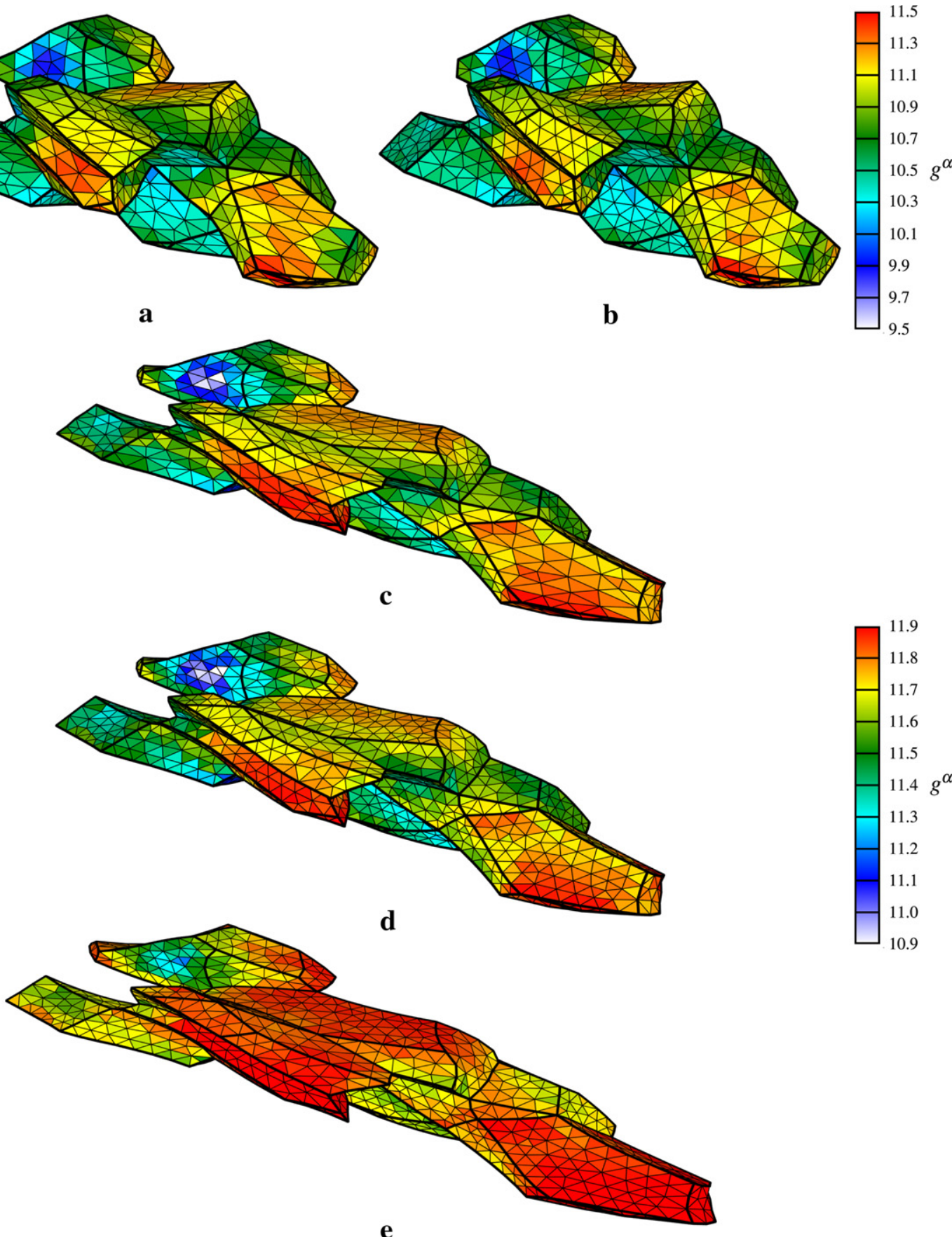

10.9

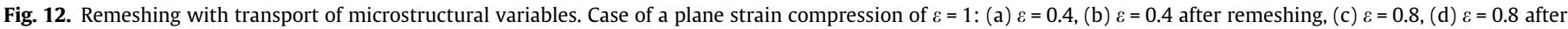
remeshing, (e) $\varepsilon=1.0$. The element colors are related to the critical resolved shear stress $g^{\alpha}$.

the projection resulting in an inadmissible geometry that is characterized by self-intersections. In these very rare cases, the old face of the mesh is copied to the new mesh. Second, the solid angle at a vertex or edge of a polyhedron can become very small as strain increases. In such a case, the element will be nearly flat on remeshing, and will degenerate even under small deformations. The present method cannot resolve this problem, which limits the maximum attainable deformation to strains between 1 and 2 , depending on the material behavior.

The second step is to transfer the microstructural and mechanical fields from the old mesh onto the new mesh. In a typical largestrain study, these fields are: the crystal orientations, the critical shear strengths on the slip systems, the strains and the stresses.
For the microstructural and strain variables, a 0-order transport is applied: each $3 \mathrm{D}$ element is assigned the values of the old element in which its centroid lies. The transport is illustrated in Fig. 12, and it can be noted that the state variables mapped relatively smoothly. Nodal values can be transfered by determining the old element in which a node resides, and then calculating its new value from the old nodal values and the element interpolation functions.

Such a 0-order transport cannot be applied to the stresses, because the new configuration would not be in mechanical equilibrium. Our alternative approach is to consider that all stresses are zero after remeshing. They are recomputed upon reloading in the subsequent finite element simulation, with the new mesh and 
$\{111\}$

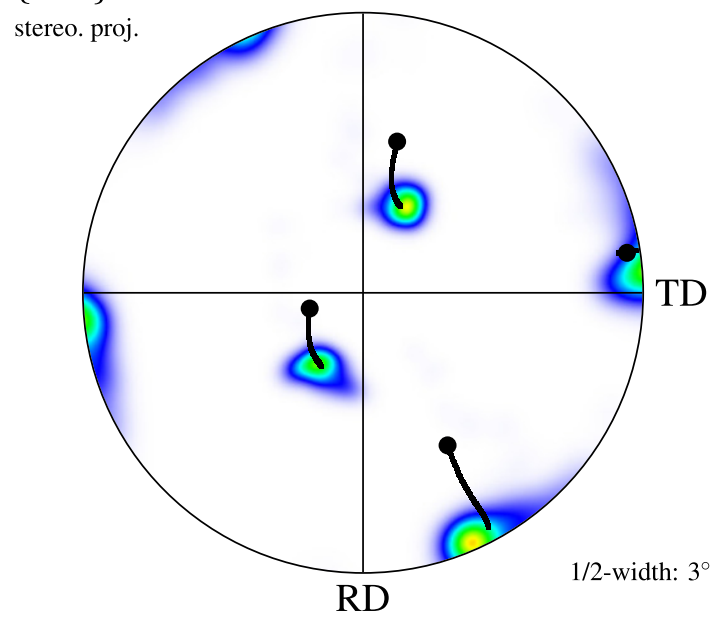

a

$\{111\}$

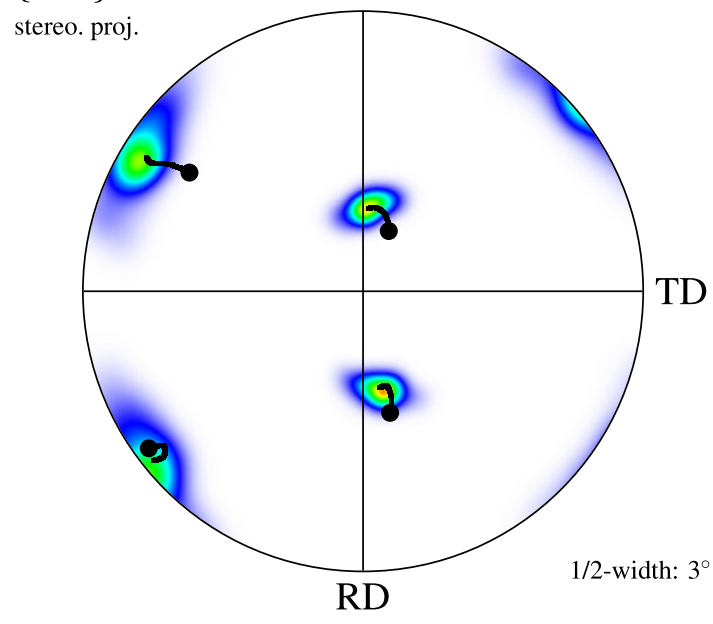

c
$\{111\}$

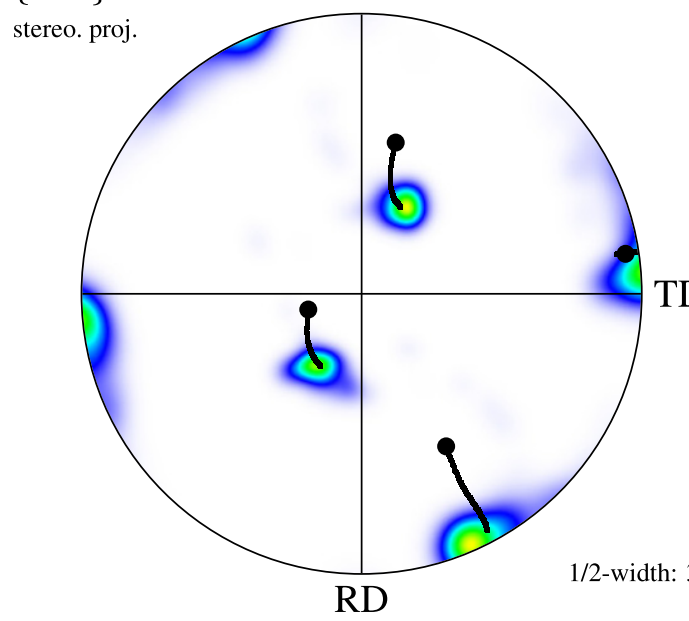

b

$\{111\}$

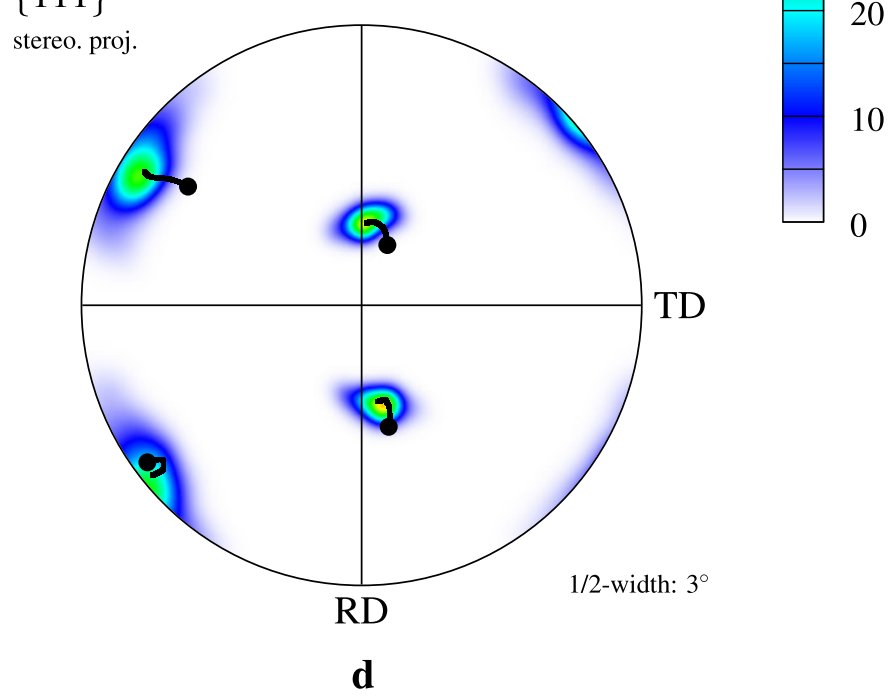

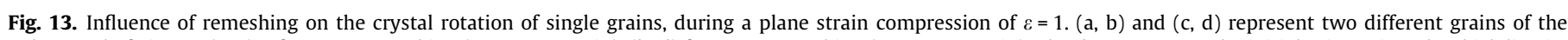

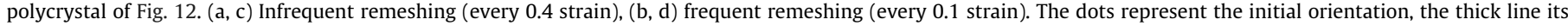
trajectory with strain, and the colour density plots the final orientation spreads.

based on the remapped microstructural variables. From the physical point of view, this corresponds to a full relaxation of the internal stresses during the remeshing, which are relative to the elastic strains. This assumption is motivated by the fact that, for the considered cases of large deformations of single phase polycrystals, the elastic strains are very small when compared to their plastic counterparts. On reloading after remeshing, the crystal stresses return to a vertex of the yield surface in an amount of strain of typically 0.002 whereas the total applied amount of plastic strain is more than two orders of magnitude bigger (about 0.5 ). The influence of this assumption is investigated by comparing two configurations that differ by the frequency of remeshing: every 0.4 strain or every 0.1 strain. This is illustrated on Fig. 13 for the case of two grains (taken from Fig. 12). No significant differences arise in either the rotation paths or final orientations. Of the nine grains investigated, the final average orientations differ only by $0.8^{\circ}$ in average, which is small compared to the rotations involved by the plastic deformation ( $16^{\circ}$ in average). This confirms that, for the case undertaken of a cubic-symmetry polycrystal, the remeshing strategy and (in particular) the assumption on the stress transfer are valid for microstructure evolution studies.

\subsection{Implementation: the Neper polycrystal generator}

The algorithms and numerical methods presented in this article have been implemented in a free (open-source) software package: Neper [36]. Neper uses as libraries the Gmsh [31] and Netgen [32] meshing packages, the libScotch mesh partitioning library [37], and the Povray ray-tracing software package [38]. Neper can be used to produce a wide variety of microstructures and mesh properties. In addition to the standard, cubic tessellations presented previously in this article, particular definitions of the domain or geometric transformations of the tessellations make it highly versatile. For example, it can be used to mesh microstructures representative of a thin sheet of recrystallized material or to account for morphological texture, as shown on Fig. 14 (a, b). Using two-dimensional distributions of polyhedron centres, one can produce columnar grains, representative of directionally-solidified 


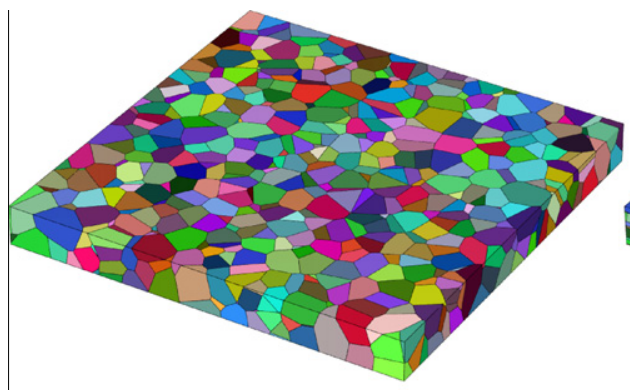

$\mathbf{a}$

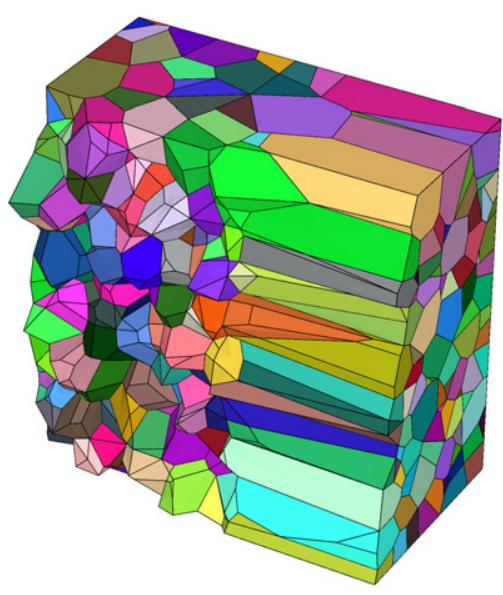

c

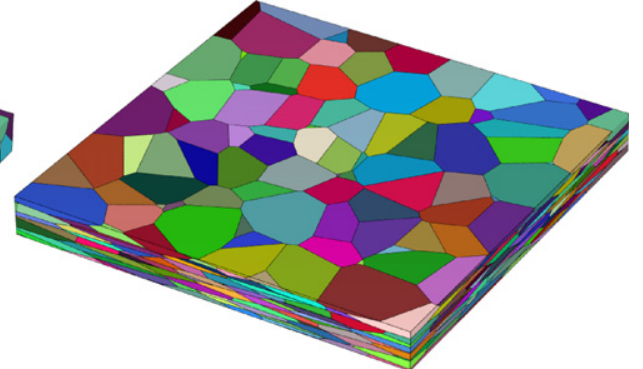

$\mathbf{b}$

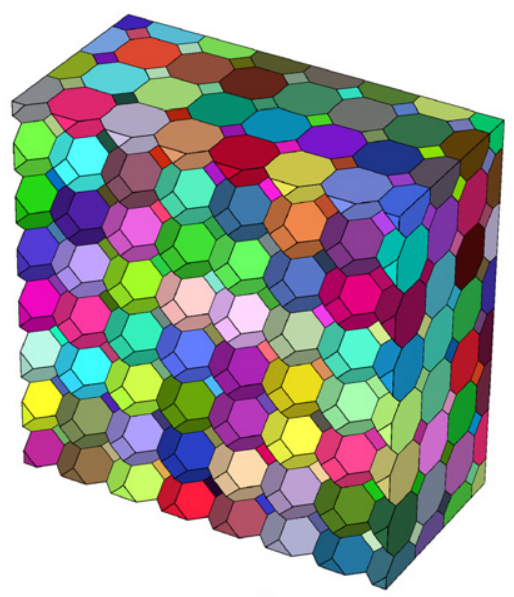

d

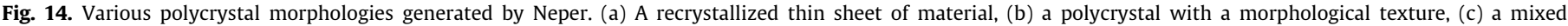

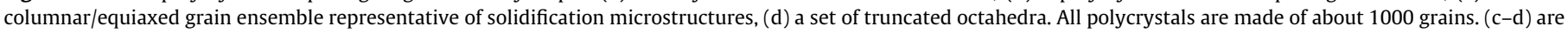
cut for the illustration.

microstructures (see Fig. 14 (c)). It may also be noticed that commonly-used, regular morphologies can be obtained by using regularly-spaced centre distributions: a cubic array provides cubes, the centres on the sites of a face-centred cubic structure provides truncated octahedra, while the body-centred structure provides dodecahedra, see Fig. 14 (d).

Different levels of discretization ( $c_{l}$ value) can easily be used, as illustrated in Fig. $15(\mathrm{a}-\mathrm{c})$. Another capability is the ability to specify different levels of discretization for different grains of the same polycrystal. This facilitate coarsening of the boundary grains, which are usually disregarded in the simulation results (due to boundary effect). An example is given in Fig. 15 (d). It is also possible to obtain different element sizes in the three directions of space, by scaling appropriately the geometry prior to meshing, and scaling back the mesh afterwards. This is particularly useful during polycrystal remeshing for large strain applications, where one may want to keep a constant spatial resolution in a given direction, as the polycrystal is deformed. This will be illustrated in Fig. 17.

Typical Neper computation times and memory needs are provided in Table 1 . Neper can readily generate and mesh random polycrystals containing several thousands of grains. The robustness of the implementation is illustrated in Fig. 16 by the meshing of a $10^{5}$-grain polycrystal.

\section{Application to texture and microtexture analysis}

Deformation textures and microtextures have been the subjects of a large number of studies in the last few decades. Texture and microtexture development is of general interest for important industrial forming operations (rolling, etc.). In particular, there has been interest around the role that textures play on the mechanical properties and subsequent thermomechanical treatments, such as phase transformations or recrystallization. The simulation by finite elements of such operations can be of major interest, but is particularly challenging because of the magnitude of the strains involved ( $\varepsilon=1$ to 2 ), which inevitably leads to excessive element distortions.

In this section, the meshing tools introduced in the preceding sections are applied to the prediction of textures and microtextures in an aluminium alloy deformed in plane strain compression to large strains $(\varepsilon=1.4)$. This example demonstrates the ability to model texture evolution over strains typical of an industrial forming process using (i) a large-scale random polycrystal (more particularly a Voronoi tessellation), and (ii) a direct free meshing/remeshing methodology. The results are compared, on the one hand, to the predictions from the Taylor model, and on the other hand, to original experimental data obtained by "microtexture tracking" $[39,40]$. The latter consists of following individual grains on an internal surface of a split sample deformed in plane strain compression. Detailed orientation measurements have been carried out by Electron Backscatter Diffraction (EBSD) on 182 grains (approximately 3000 measurements per grain), at the successive (logarithmic) strains of $\varepsilon=0,0.42,0.77$ and 1.2 , enabling detailed analyses.

\subsection{Simulation}

The simulation is carried out on the 3000-grain polycrystal illustrated on Fig. 1. The polycrystal has initial grain orientations randomly chosen from a uniform distribution. The polycrystal is subjected to plane strain compression to a strain $\varepsilon=1.4$ (75\% thickness reduction). The deformed microstructure is illustrated in Fig. 17. Remeshing was applied at $\varepsilon=0.4,0.8$, and 1.0. Orthotropic meshing was used, so that the element density remained constant 


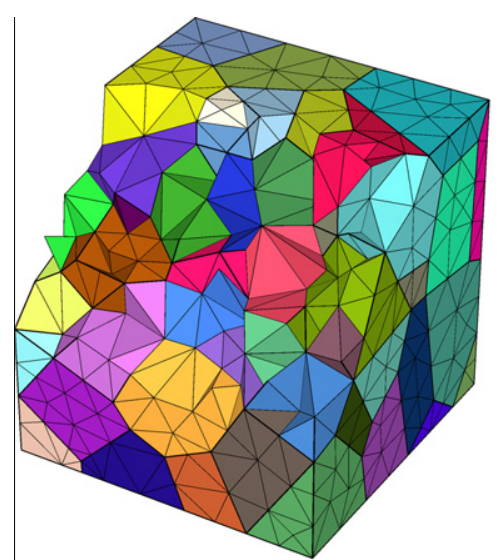

$\mathbf{a}$

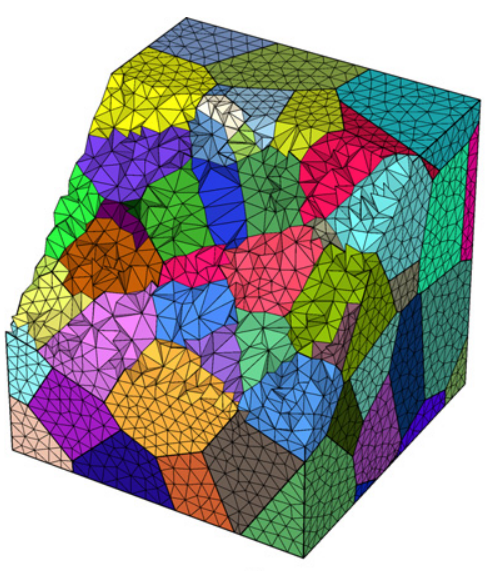

b

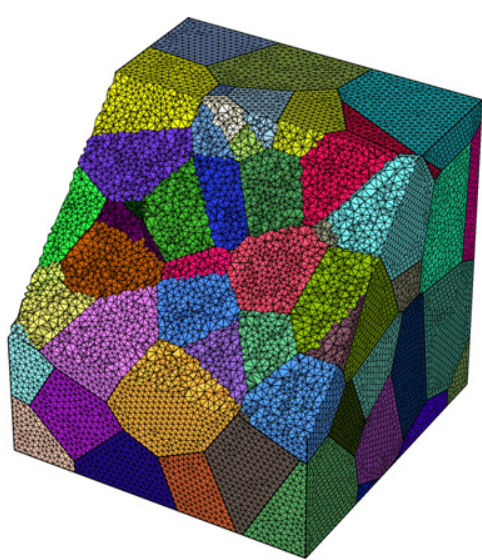

c

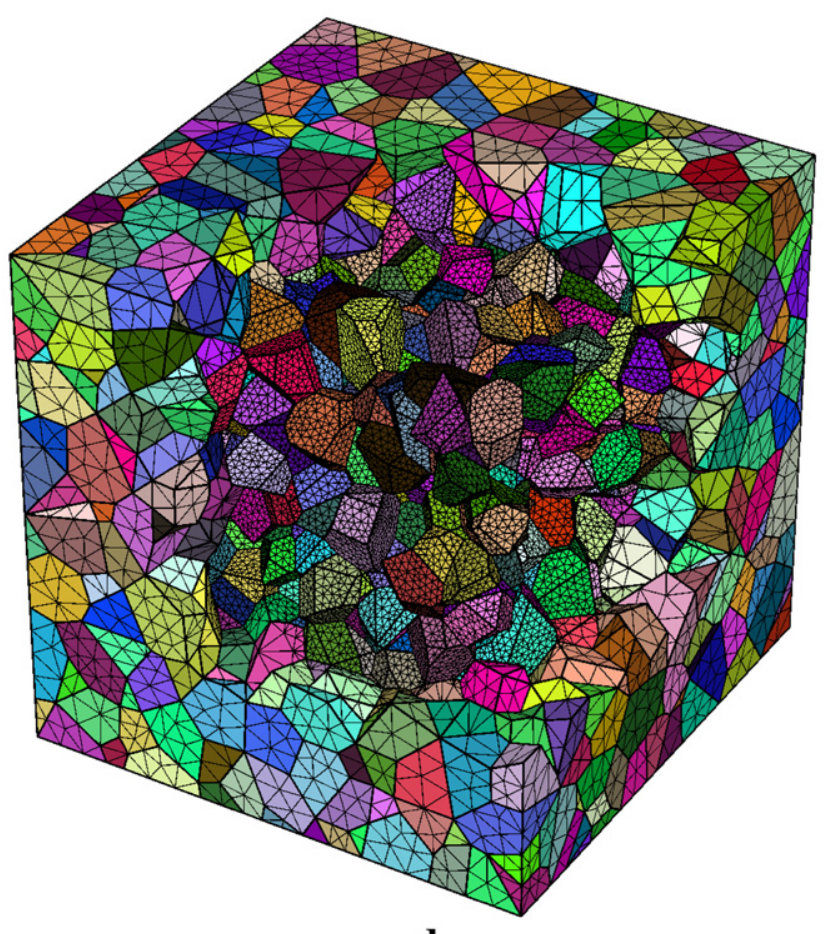

d

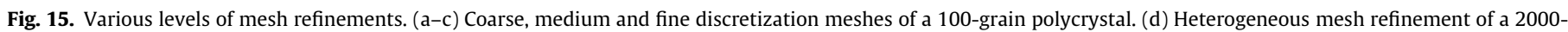
grain polycrystal: inner grains with fine meshes and outer grains with coarse meshes.

in the compression and transverse directions, while the element size remained constant in the rolling direction (this can be noticed on Fig. 17 by a mesh denser in the compression direction than in the other directions). The mesh was comprised of 407,000 10-node elements $/ 563,000$ nodes at the beginning of the simulation $(\varepsilon=0)$ and 973,000 elements/1,336,000 nodes at the end $(\varepsilon=1.4)$.

The plastic deformation is accommodated by slip on specific crystallographic systems. The twelve octahedral $\{111\}\langle 110\rangle$ systems are considered to be potentially active (FCC crystal structure). Slip is assumed to follow a viscoplastic behavior, where the slip rate $\dot{\gamma}^{\alpha}$ on a system $\alpha$ is related to the resolved shear stress $\tau^{\alpha}$ through the power law:

$\dot{\gamma}^{\alpha}=\dot{\gamma}_{0}\left|\frac{\tau^{\alpha}}{g^{\alpha}}\right|^{\frac{1}{m}} \operatorname{sgn}\left(\tau^{\alpha}\right)$

with

$\dot{g}^{\alpha}=h_{0}\left(\frac{g_{s}-g^{\alpha}}{g_{s}-g_{0}}\right) \dot{\gamma} \quad$ where $\dot{\gamma}=\sum_{\alpha}\left|\dot{\gamma}^{\alpha}\right|$
By definition, $\operatorname{sgn}(x)=1$ if $x \geqslant 0$ and -1 if $x<0$. The numerical values of the material parameters have been derived from the experimental measurements: $\dot{\gamma}_{0}=1, m=0.12, h_{0}=4 \mathrm{MPa}, g_{0}=8 \mathrm{MPa}$, and $g_{s}=12 \mathrm{MPa}$. At $\varepsilon=0$, all $g^{\alpha}$ are taken equal to $g_{0}$. In such a case, it can be noticed from Eq. (9) that all $g^{\alpha}$ increase equally with strain.

A complete description of the constitutive model and the FEM implementation can be found in Refs. [4,5]. The full simulation, including the initial meshing, the remeshings, and the FEM solutions (run on 128 cores of a cluster running the Linux kernel), took $100 \mathrm{~h}$. In the analysis, only the inner grains of the polycrystal are taken into consideration to avoid boundary effects (1972 grains).

\subsection{Textures}

The FEM texture obtained at $\varepsilon=1.2$ is compared to the experimental data and to the Taylor model predictions in Fig. 18. The textures are described by orientation distribution functions represented over a fundamental region of Rodrigues' space [41]. 


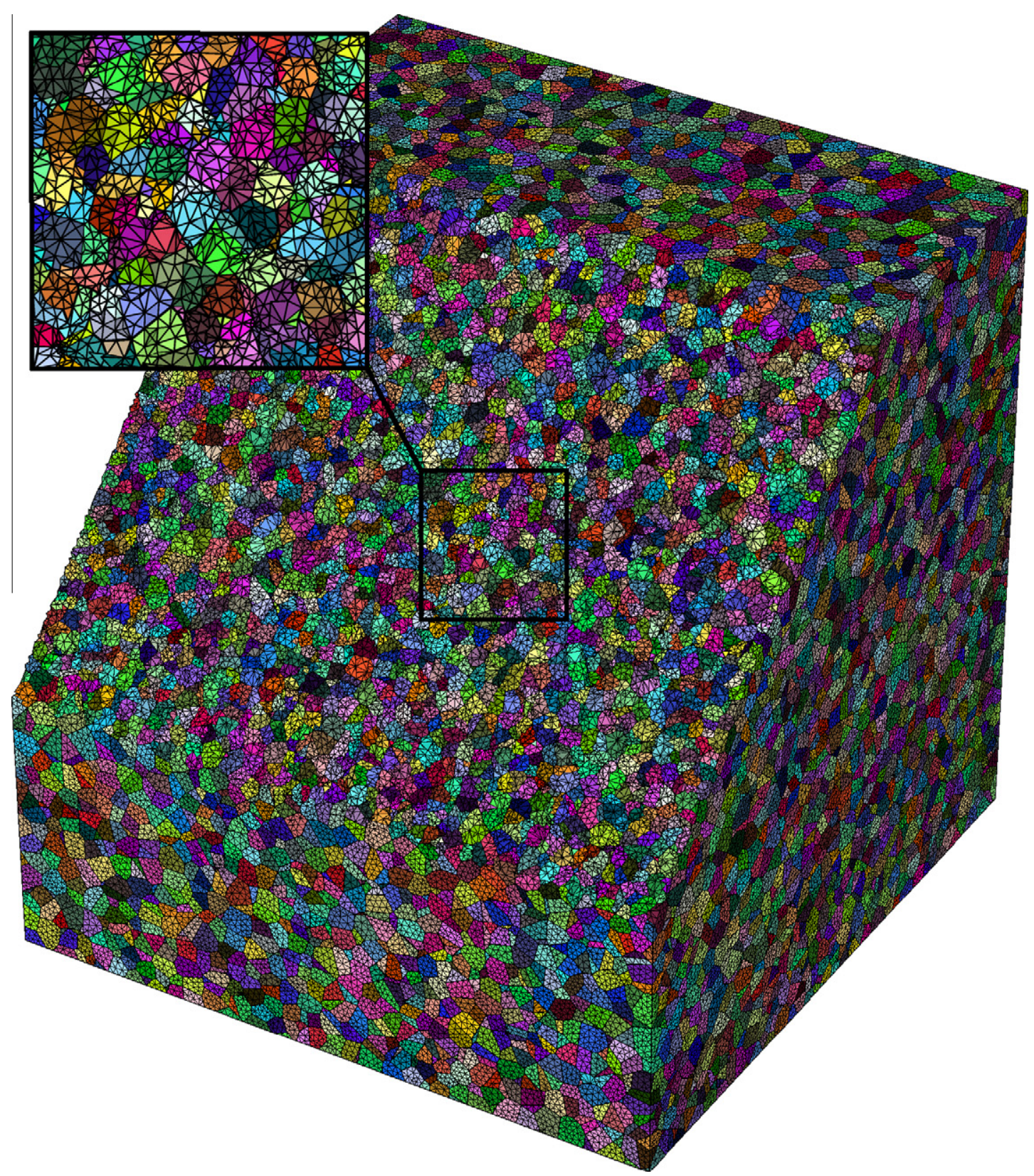

Fig. 16. Free meshing of a $10^{5}$-grain Voronoi tessellation. The mesh contains $19,600,000$ 10-node elements and $26,400,000$ nodes.

The experimental and simulated textures show a well-developed $\beta$-fiber, characteristic of FCC metals deformed in plane strain compression $[42,43]$. The Taylor model overestimates significantly the average intensity of the texture. Particularly, it overestimates the intensity of the Copper component and underestimates the intensity of the Brass component, a typical observation [42,43]. The finite element formulation predicts a weaker texture with respect to the Taylor predictions, thus providing better agreement with the experiments. Similar observations have been made from level-set simulations [19].

\subsection{In-grain orientation spreads}

The crystal rotations can also be compared at grain level. In this section, we focus on the orientation distributions, or spreads, developed within the individual grains (the Taylor model does not produce such distributions, and so will not be considered in the following). In Fig. 19, an experimental and an FEM in-grain orientation spread are illustrated for two grains of approximately the same final orientation $(\varepsilon=1.2)$. The comparison illustrates the ability of the finite element method for reproducing trends observed in the experiments.

Before going further, it should be noted that the FEM polycrystal reproduces the experimental one only in a statistical sense (it exhibits similar metrics for the distributions of lattice orientation, grain shape and grain size), which does not allow a grain-by-grain comparison. As a consequence, in the following the experimental and simulated in-grain orientation spreads will be compared only in terms of distribution over all grains.

An orientation spread can be described by several metrics associated with the moments of a distribution. The average size (mean) is deduced from the average disorientation angle with respect to the average orientation. The average orientation is calculated by quaternion averaging [44-47] and the disorientations are then calculated in the standard manner by taking the cubic crystal symmetry into account. Implementations are provided in Orilib [48]. The 


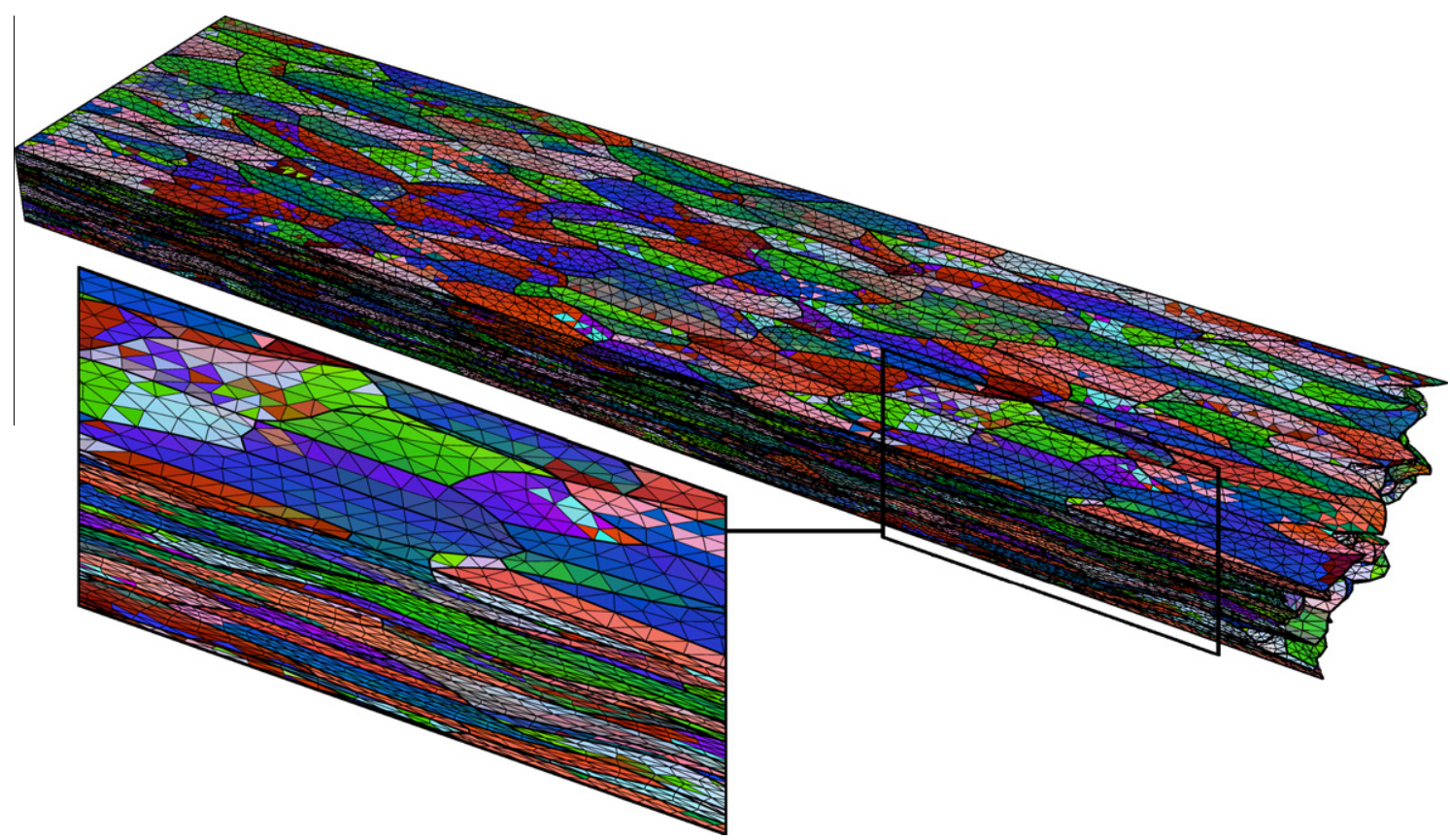

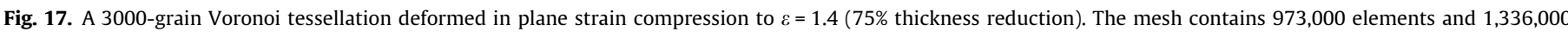

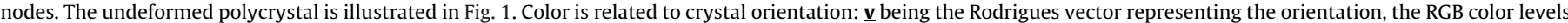
are $255 \times\left(v_{i}+\sqrt{2}-1\right) /[2 \times(\sqrt{2}-1)]$.

Table 1

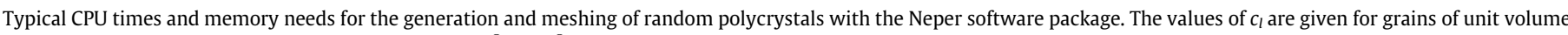
in average. Simulations performed on a $2.40 \mathrm{GHz}$ Intel ${ }^{\circledR}$ Xeon ${ }^{\circledR}$ processor of a computer running the Linux kernel.

\begin{tabular}{|c|c|c|c|c|c|c|}
\hline \multirow[t]{2}{*}{ Grain number } & \multicolumn{3}{|c|}{ Meshing with $c_{l}=0.50$} & \multicolumn{3}{|c|}{ Meshing with $c_{l}=0.25$} \\
\hline & CPU (sec.) & RAM (Mb) & Elts/Nodes $(\times 1000)$ & CPU (sec.) & RAM (Mb) & Elts/Nodes $(\times 1000)$ \\
\hline 100 & 43 & 20 & $18 / 27$ & 68 & 35 & $65 / 93$ \\
\hline 300 & 113 & 33 & $58 / 82$ & 209 & 78 & $190 / 269$ \\
\hline 1000 & 444 & 82 & $194 / 270$ & 790 & 230 & $639 / 887$ \\
\hline 3000 & 1840 & 219 & $582 / 798$ & 3006 & 658 & $1907 / 2621$ \\
\hline 10000 & 7350 & 1455 & $1953 / 1455$ & 17160 & 2142 & $6332 / 8640$ \\
\hline
\end{tabular}

distributions of the average disorientations over all grains are illustrated on Fig. 20. While the average disorientations are zero at $\varepsilon=0$, they have experimental values of $5.1^{\circ}, 6.4^{\circ}$, and $7.0^{\circ}$ at $\varepsilon=0.42,0.77$ and 1.2 , respectively. That is, they develop quickly at the beginning of the deformation, then tend to stabilize. The finite element method provides the same tendencies, with average values of $4.1^{\circ}, 5.5^{\circ}$ and $6.5^{\circ}$, successively, and distributions similar to the experimental ones.

Further comparisons between experiment and simulation are provided with the correlation between the orientation spreads that an individual grain can show from one strain level to the next. They are illustrated in Fig. 21. For both the experimental and FEM cases, the individual orientation spreads tend to increase as deformation increases, with quite strong correlations from one deformation to the next. The correlations can be quantified using linear correlation coefficients, a value of 0 denoting no correlation and a value of 1 denoting a perfect correlation. From $\varepsilon=0.42$ to 0.77 , they have values of 0.78 and 0.82 , for the experiments and FEM, respectively. From $\varepsilon=0.77$ to 1.2 , they have values of 0.88 and 0.89 , respectively. The size evolution of the orientation spreads obtained by FEM is consequently in good agreement with the experiments.

\section{Discussion}

Accurate predictions of the local mechanical fields developing in polycrystalline materials require the use of representative polycrystal morphologies. In the last few decades, experimental and numerical efforts have been made to characterize and reproduce the main morphological properties of polycrystalline microstructures $[1-3,6,16-20]$. From any polycrystal morphology, material behavior and boundary conditions, the finite element method coupled with a free meshing technique provides the capability to determine accurately the mechanical and microstructural fields which develop in the polycrystal.

The present work aims at providing a complete and robust methodology for the generation of high-quality meshes of largescale Voronoi tessellations, that is, comprising several thousand of grains. The method consists of removing the small edges of the geometry, thereby eliminating the features responsible to mesh overrefinement. This operation leads to local geometry distortions: the boundary of the faces (made of the vertices and edges) can leave their original planes, and consequently an interpolation method is needed to define their interiors. A simple remedy is proposed which consists in dividing such a face into triangle parts, allowing the distortion of the face to be quantified by its "flatness fault" (the maximum angle between the normal at two locations of the face). The algorithm tends to minimize the geometry distortions, so that the face flatness faults are retained under a given threshold value (typically $20^{\circ}$ ). This method avoids the local mesh overrefinements and ensures element quality. It should also be noted that geometry regularization improved the morphological properties of the polycrystal: the 

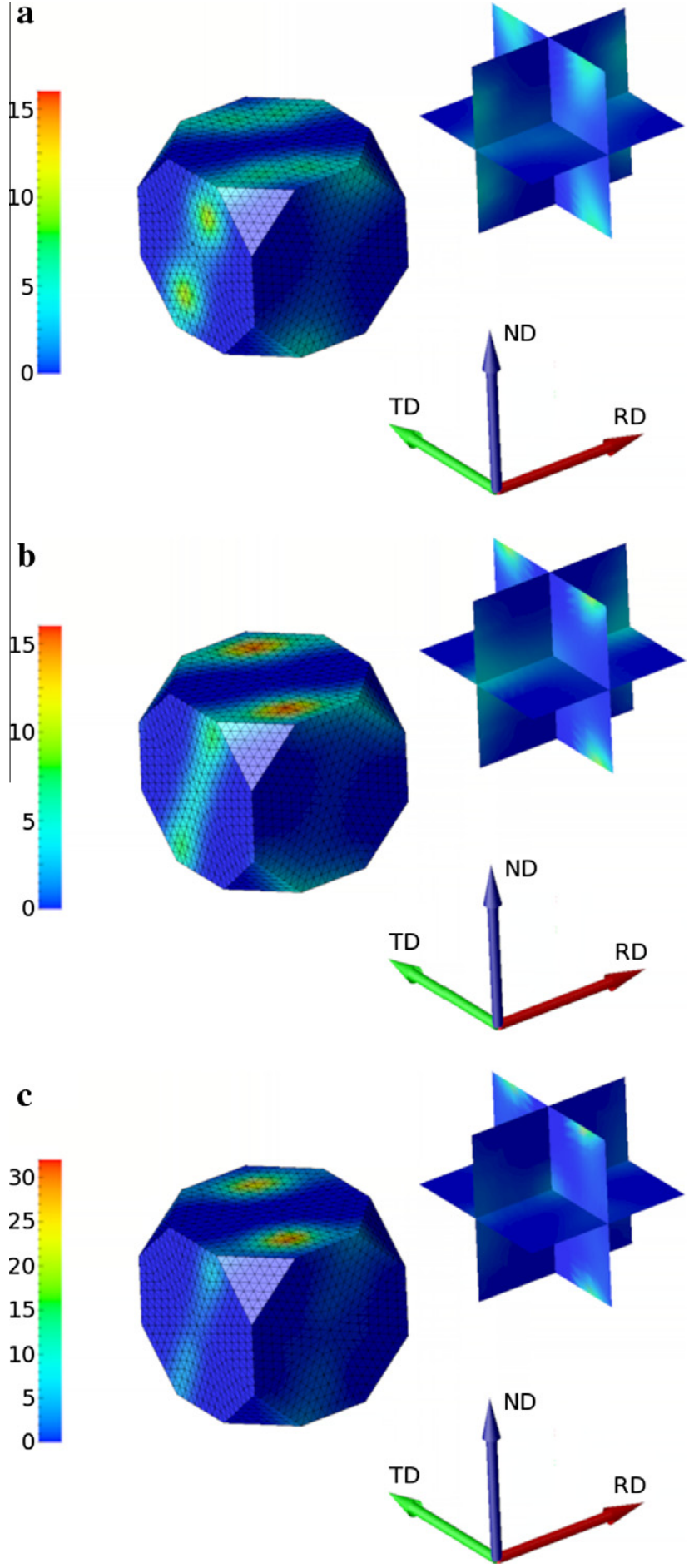

Fig. 18. Macrotextures in an aluminium alloy deformed in plane strain compression. (a) Experiments, (b) finite element model, (c) Taylor model. Note the different color scales between ( $a$ and $b$ ) versus (c). The positions in Rodrigues' space of the $\beta$ fiber components are: $S=(0.254,-0.235,-0.291), \quad(-0.254,0.235,0.291)$, $(-0.254,-0.235,0.291)$ and $(0.254,0.235,0.291) ; \quad$ Brass $=(-0.414,-0.132,0.318)$ and $(0.414,0.132,0.318)$; Copper $=(-0.132,-0.318,0.414)$ and $(0.132,0.318,0.414)$ [41].

average number of grain first neighbors, typically overestimated by Voronoi tessellations (value of 15 ), can be significantly reduced, and even made coincide with the experimental values (between 12 and 14). Changes in tessellation topology are also involved: more than four grains can intersect at a single vertex and more than three faces can intersect at a single edge.
To ensure meshing robustness and, at the same time, improve element quality needed for large strain applications, an additional, very pragmatic approach has been introduced, called "multimeshing". It is motivated by the fact that a number of $2 \mathrm{D}$ and $3 \mathrm{D}$ meshing software packages are available to the user, each with its own strengths and weaknesses. Multimeshing consists of meshing each entity (face or volume) with several meshers, and then using the mesh of best quality. This gives improved mesh quality of the whole polycrystal (see Fig. 11).

The meshes obtained with the meshing and multimeshing strategies have been used in simulations to reach strains of the order 0.5 , which is close to what can be attained with "perfect" meshes (regular morphologies meshed into regular tetrahedral elements), typically 0.7 [10]. Applications such as industrial forming operations involve strains of the order $1-2$, which as a consequence cannot be attained with a single polycrystal mesh. To solve this problem, a remeshing strategy has been presented, which includes mesh regeneration and data remapping onto the new mesh. The mesh generation itself is very similar to the meshing of undeformed regularized Voronoi tessellations. Concerning data remapping, a 0-order transport has been proposed: the values at a given element of the new mesh are taken from the element of the old mesh in which its centroid lies. Higher-order schemes have been deployed in the context of adaptive Lagrangian Eulerian (ALE) codes [49]. Such a transfer methodology cannot be applied to stress, because the new configuration would not be in mechanical equilibrium. A simple method has been employed, which consists of setting all stresses equal to zero after remeshing. The stresses are recomputed in the subsequent finite element simulation, with the new mesh and based on the remapped microstructural variables. The influence of remeshing has been evaluated quantitatively by comparing the crystal rotations of individual grains between simulations having more or less frequent remeshing (every 0.4 strain against every 0.1 strain). Of the nine grains investigated, only a minor influence has been noticed on the average orientations at $\varepsilon=1$ (disorientation $<0.8^{\circ}$ ), as well as on the in-grain orientation spreads (see Fig. 13). Thus, the presented remeshing approach proved effective not only for the prediction of textures, but also for the in-grain orientation spreads at large strains.

All the methods presented in this study has been implemented into a free (open-source) software package: Neper [36]. Examples of meshes have illustrated the ability and robustness of the proposed methodology and its implementation for generating high-quality meshes of large polycrystals. Typical CPU times and memory needs have been provided, which show that the polycrystal generation and meshing are much faster than the FEM simulation itself.

To demonstrate the capabilities of the polycrystal generation and meshing methodology, a large-strain application has been presented: the simulation of texture and microtexture development in an aluminium polycrystal submitted to plane strain compression. It combines: (i) a large-scale Voronoi tessellation (3000 grains), (ii) strain levels representative of industrial forming operations $(\varepsilon=1.4$ ), and (iii) direct free meshing/remeshing strategies for a conventional FEM solution. The simulation has been shown to reproduce satisfactorily experimental data on the textures and, more interestingly, on the microtexture properties. In particular the size and evolution of the in-grain orientation spreads are shown to compare well.

\section{Conclusions}

A methodology for the generation and good-quality meshing of large-scale random polycrystals have been presented. The polycrystals are represented by Voronoi tessellations. The latter have 
a $\{111\}$

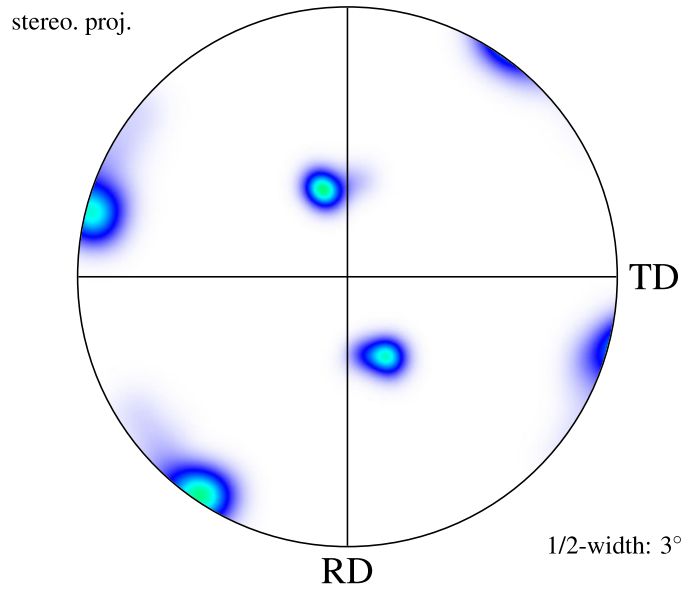

b $\{111\}$

stereo. proj.

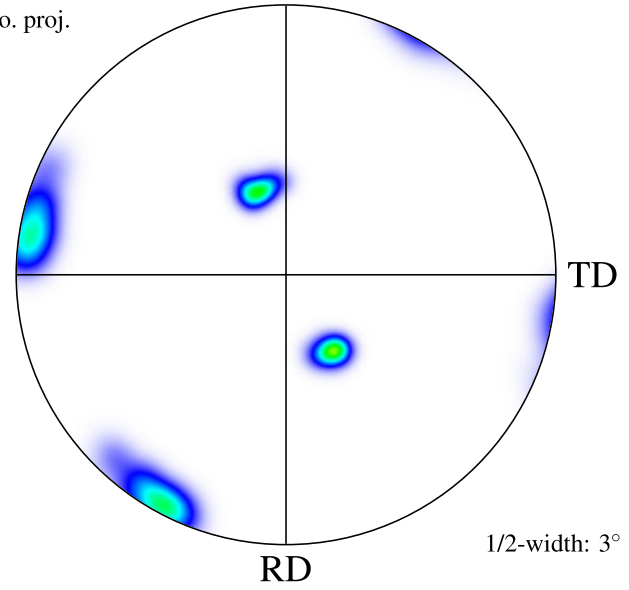

80

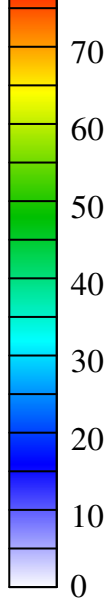

Fig. 19. Examples of (a) experimental and (b) finite element in-grain orientation spreads at $\varepsilon=1.2$, for two grains of approximately the same average orientation.
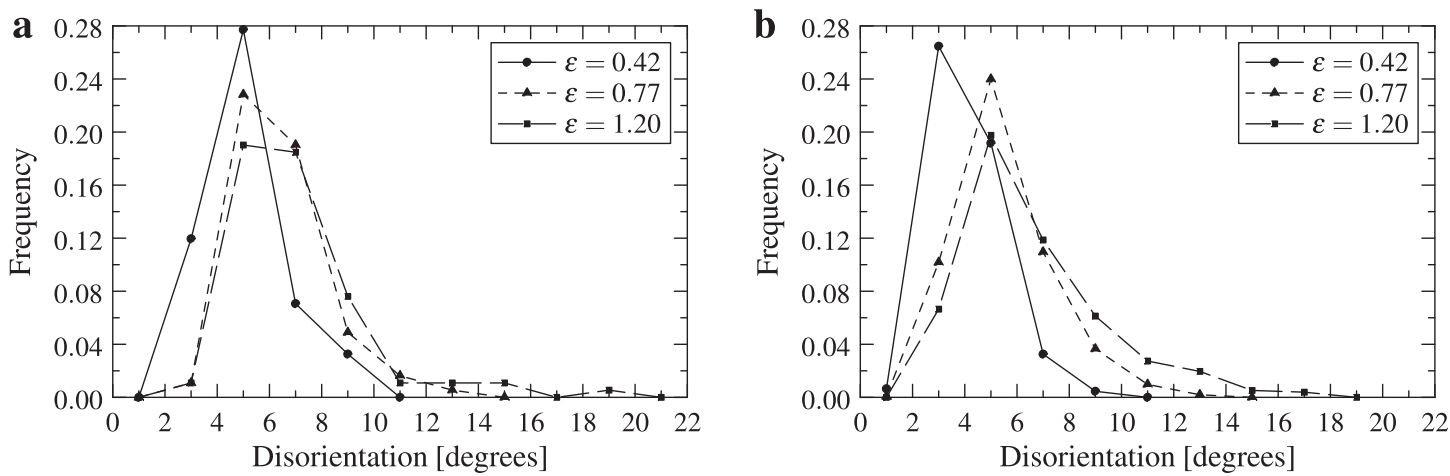

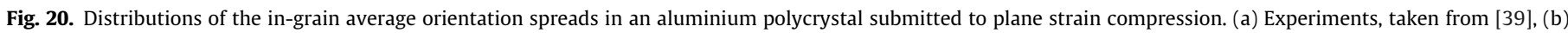
finite elements. Note that only the grains that show unimodal rotations as described in Ref. [39] are considered.
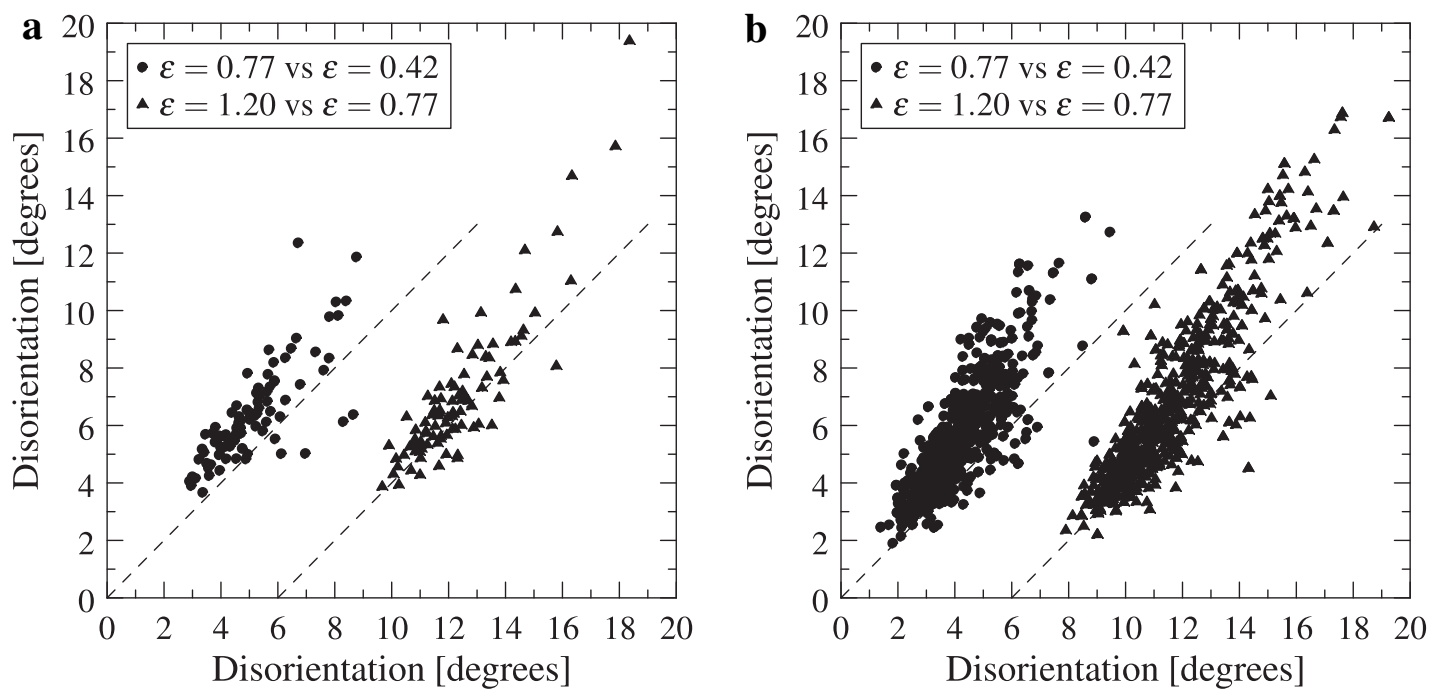

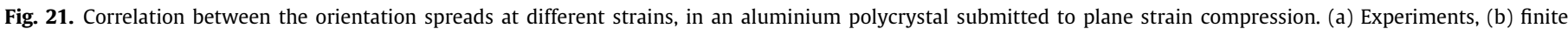
elements. The right-hand side data clouds have been shifted $6^{\circ}$ along the $x$-axis for clarity.

been shown to include a large number of geometrical details, which make it particularly challenging to render a mesh of good- quality elements, especially when the number of grains becomes large (several thousand). Different, complementary strategies have 
been merged to solve this problem. Geometry regularization diminishes the number of small features of the geometry (edges and faces), which are problematic for meshing. It also improves the morphological properties of the polycrystal with regard to real polycrystal morphologies, notably by decreasing the average number of grain first neighbors. Once the geometry has been regularized, the multimeshing strategy constructs a mesh simultaneously using several meshing algorithms. Finally, a remeshing method is implemented for large strain applications (strains of 1-2). The presented methods and algorithms have enabled us to generate a wide variety of microstructures. The robustness of the methodology and its implementation in the free (open-source) software package Neper are illustrated by a texture and microtexture simulation study involving a 3000-grain Voronoi tessellation deformed up to a strain of $\varepsilon=1.4$.

\section{Acknowledgments}

One of the author (R.Q.) wishes to acknowledge the developers of the different programs and libraries used by Neper, and in particular Ch. Geuzaine (Gmsh mesher [31]) and F. Pellegrini (libScotch mesh partitioner [37]), for making their programs freely available, and for the punctual help they have provided. The authors thank the reviewers for providing particularly detailed reports, which contributed to improve this article. Partial support has been provided by ONR as R.Q. was a postdoctoral associate at Cornell University, under Grant No. N00014-09-1-0447 and N00014-05-1-0506.

\section{References}

[1] C. Zhang, A. Suzuki, T. Ishimaru, M. Enomoto, Characterization of threedimensional grain structure in polycrystalline iron by serial sectioning, Metal Mater. Trans. A 35A (2004) 1927-1953.

[2] K. Döbrich, C. Rau, C. Krill III, Quantitative characterization of the threedimensional microstructure of polycrystalline Al-Sn using X-ray microtomography, Metal. Mater. Trans. A 35A (2004) 1953-1961.

[3] D. Rowenhorst, A. Lewis, G. Spanos, Three-dimensional analysis of grain topology and interface curvature in a $\beta$-titanium alloy, Acta Mater. 58 (2010) 5511-5519.

[4] E.B. Marin, P.R. Dawson, On modelling the elasto-viscoplastic response of metals using polycrystal plasticity, Comput. Methods Appl. Mech. Engrg. 165 (1998) 1-21.

[5] E.B. Marin, P.R. Dawson, Elastoplastic finite element analyses of metal deformations using polycrystal constitutive models, Comput. Methods Appl. Mech. Engrg. 165 (1998) 23-41.

[6] F. Barbe, L. Decker, D. Jeulin, G. Cailletaud, Intergranular and intragranular behavior of polycrystalline aggregates. Part 1: F.E. model, Int. J. Plast. 17 (4) (2001) 513-536.

[7] F. Barbe, S. Forest, G. Cailletaud, Intergranular and intragranular behavior of polycrystalline aggregates. Part 2: Results, Int. J. Plast. 17 (4) (2001) 537-563.

[8] G.B. Sarma, P.R. Dawson, Effects of interactions among crystals on the inhomogeneous deformations of polycrystals, Acta Mater. 44 (5) (1996) 1937-1953.

[9] D. Raabe, Z. Zhao, W. Mao, On the dependence of in-grain subdivision and deformation texture of aluminum on grain interaction, Acta Mater. 50 (17) (2002) 4379-4394.

[10] Z. Zhao, S. Kuchnicki, R. Radovitzky, A. Cuitiño, Influence of in-grain mesh resolution on the prediction of deformation textures in fcc polycrystals by crystal plasticity FEM, Acta Mater. 55 (7) (2007) 2361-2373.

[11] H. Ritz, P.R. Dawson, Sensitivity to grain discretization of the simulated crystal stress distributions in FCC polycrystals, Model. Simul. Mater. Sci. Engrg. 17 (2009) 1-21.

[12] F. Barbe, R. Quey, A. Musienko, G. Cailletaud, Three-Dimensiona characterization of strain localization bands in a high-resolution elastoplastic polycrystal, Mech. Res. Commmun. 36 (2009) 762-768.

[13] T. Kanit, S. Forest, I. Galliet, V. Mounoury, D. Jeulin, Determination of the size of the representative volume element for random composites: statistical and numerical approach, Int. J. Solids Struct. 40 (13-14) (2003) 3647-3679.

[14] O. Diard, S. Leclercq, G. Rousselier, G. Cailletaud, Evaluation of finite element based analysis of 3D multicrystalline aggregates plasticity: application to crystal plasticity model identification and the study of stress and strain fields near grain boundaries, Int. J. Plast. 21 (4) (2005) 691-722.

[15] R. Lebensohn, M. Montagnat, P. Mansuy, P. Duval, J. Meysonnier, A. Philip, Modeling viscoplastic behavior and heterogeneous intracystalline deformation of culumnar ice polycrystals, Acta Mater. 57 (2009) 1405-1415.
[16] F. Barbe, R. Quey, A numerical modelling of 3D polycrystal-to-polycrystal diffusive phase transformations involving crystal plasticity, Int. J. Plast. uncorrected proofs, doi: <doi:10.1016/j.ijplas.2010.09.008>.

[17] F. Wakai, N. Enomoto, H. Ogawa, Three-dimensional microstructural evolution in ideal grain growth - General statistics, Acta Mater. 48 (2000) 1297-1311.

[18] C. Krill III, L.-Q. Chen, Computer simulation of 3D grain growth using a phasefield model, Acta Mater. 50 (2002) 3057-3073.

[19] H. Resk, L. Delannay, M. Bernacki, T. Coupez, R. Logé, Adaptative mesh refinement and automatic remeshing in crystal plasticity finite element simulations, Model. Simul. Mater. Sci. Engrg. 17 (2009) 1-22.

[20] A. Brahme, M. Alvi, D. Saylor, J. Fridy, A. Rollett, 3D reconstruction of microstructure in a commercial purity aluminum, Scr. Mater. 55 (2006) 75-80.

[21] R. Lebensohn, N-site modeling of a 3D viscoplastic polycrystal using fast Fourier transform, Acta Mater. 49 (2001) 2723-2737.

[22] Z. Zhao, M. Ramesh, D. Raabe, A. Cuitiño, R. Radovitzky, Investigation of threedimensional aspects of grain-scale plastic surface deformation of an aluminum oligocrystal, Int. J. Plast. 24 (2008) 2278-2297.

[23] Y. Wei, L. Anand, Grain-boundary sliding and separation in polycrystalline metals: application to nanocrystalline fcc metals, J. Mech. Phys. Solids 52 (2004) 2587-2616.

[24] N. Osipov, A. Gourgues Lorenzon, B. Marini, V. Mounoury, F. Nguyen, G. Cailletaud, FE modelling of bainitic steels using crystal plasticity, Phil. Mag. 88 (2008) 3757-3777.

[25] A. Musienko, G. Cailletaud, Simulation of inter- and transgranular crack propagation in polycrystalline aggregates due to stress corrosion cracking, Acta Mater. 57 (2009) 3840-3855.

[26] E. Gilbert, Random subdivisions of space into crystals, Ann. Math. Stat. 33 (1962) 958-972.

[27] M. Nygårds, P. Gudmundson, Three-dimensional periodic Voronoi grain models and micromechanical FE-simulations of a two-phase steel, Comput. Mater. Sci. 24 (4) (2002) 513-519.

[28] F. Fritzen, T. Böhlke, E. Schnack, Periodic three-dimensional mesh generation for crystalline aggregates based on Voronoi tessellations, Comput. Mech. 43 (2009) 701-713

[29] C. Gérard, B. Bacroix, M. Bornert, G. Cailletaud, J. Crépin, S. Leclercq, Hardening description for FCC materials under complex loading paths, Comput. Mater. Sci. 45 (2009) 751-755.

[30] S. Kumar, S.K. Kurtz, Monte-Carlo study of angular and edge length distributions in a three-dimensional Poisson-Voronoi tesselation, Mater. Charact. 34 (1) (1995) 15-27.

[31] C. Geuzaine, J.-F. Remacle, Gmsh: a three-dimensional finite element mesh generator with built-in pre- and post-processing facilities, Int. J. Numer. Methods Engrg. 79 (2009) 1309-1331.

[32] J. Schoberl, Netgen, an advancing front 2D/3D-mesh generator based on abstract rules, Comput. Visual. Sci. 52 (1997) 1-41.

[33] L. Delannay, M.A. Melchior, J.W. Signorelli, J.-F. Remacle, T. Kuwabara, Influence of grain shape on the planar anisotropy of rolled steel sheets Evaluation of three models, Comput. Mater. Sci. 45 (2009) 739-743.

[34] J. Pascal, P.-L. George, Mesh Generation, Hermes Science Publishing, 2000.

[35] J. Shewchuk, Triangle: Engineering a 2D Quality Mesh Generator and Delaunay Triangulator, Applied Computational Geometry: Towards Geometric Engineering, Lecture Notes in Computer Science 1148 (1996) 203-222.

[36] Neper: a 3D random polycrystal generator for the finite element method (version 1.9), <http://neper.sourceforge.net>.

[37] F. Pellegrini, Scotch and libScotch 5.1 User's Guide, INRIA Bordeaux Sud-Ouest, ENSEIRB \& LaBRI, UMR CNRS 5800, 2008.

[38] Povray: the persistence of vision raytracer, <http://www.povray.org>.

[39] R. Quey, D. Piot, J.H. Driver, Microtexture tracking in hot-deformed polycrystalline aluminium: experimental results, Acta Mater. 58 (2010) 1629-1642.

[40] R. Quey, D. Piot, J.H. Driver, Microtexture tracking in hot-deformed polycrystalline aluminium: comparison with simulations, Acta Mater. 58 (2010) 2271-2281.

[41] A. Kumar, P.R. Dawson, Computational modeling of f.c.c. deformation textures over Rodrigues' space, Acta Mater. 48 (10) (2000) 2719-2736

[42] C. Maurice, J.H. Driver, Hot rolling textures of f.c.c. metals - Part I. Experimental results on Al single and polycrystals, Acta Mater. 45 (11) (1997) 4627-4638.

[43] C. Maurice, J.H. Driver, Hot rolling textures of f.c.c. metals - Part II. Numerical simulations, Acta Mater. 45 (11) (1997) 4639-4649.

[44] N.C. Krieger Lassen, D.J. Jensen, K. Conradsen, On the statistical analysis of orientation data, Acta Crystall. A50 (1994) 741-748.

[45] M. Humbert, N. Gey, J. Muller, C. Esling, Determination of a mean orientation from a cloud of orientations. Application to electron back-scattering pattern measurements, J. Appl. Crystallogr. 26 (1996) 662-666.

[46] F.J. Humphreys, P.S. Bate, P.J. Hurley, Orientation averaging of electron backscattered diffraction data, J. Microstruc. 201 (2001) 50-58.

[47] J.-C. Glez, J.H. Driver, Orientation distribution analysis in deformed grains, J. Appl. Crystallogr. 34 (2001) 280-288.

[48] Orilib: a collection of routines for orientation manipulation (version 2.0), $<$ http://orilib.sourceforge.net>.

[49] D.J. Benson, Momentum advection on unstructured staggered quadrilateral meshes, Int. J. Numer. Methods Engrg. 75 (2008) 1549-1580. 